\title{
REVIEWS
}

\section{Combining epigenetic drugs with other therapies for solid tumours - past lessons and future promise}

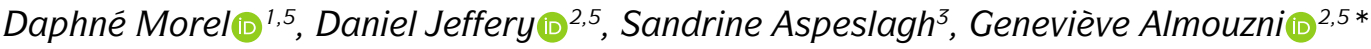 \\ and Sophie Postel-Vinay (10) ${ }^{1,4,5 *}$
}

Abstract | Epigenetic dysregulation has long been recognized as a key factor contributing to tumorigenesis and tumour maintenance that can influence all of the recognized hallmarks of cancer. Despite regulatory approvals for the treatment of certain haematological malignancies, the efficacy of the first generation of epigenetic drugs (epi-drugs) in patients with solid tumours has been disappointing; however, successes have now been achieved in selected solid tumour subtypes, thanks to the development of novel compounds and a better understanding of cancer biology that have enabled precision medicine approaches. Several lines of evidence support that, beyond their potential as monotherapies, epigenetic drugs could have important roles in synergy with other anticancer therapies or in reversing acquired therapy resistance. Herein, we review the mechanisms by which epi-drugs can modulate the sensitivity of cancer cells to other forms of anticancer therapy, including chemotherapy, radiation therapy, hormone therapy, molecularly targeted therapy and immunotherapy. We provide a critical appraisal of the preclinical rationale, completed clinical studies and ongoing clinical trials relating to combination therapies incorporating epi-drugs. Finally, we propose and discuss rational clinical trial designs and drug development strategies, considering key factors including patient selection, tumour biomarker evaluation, drug scheduling and response assessment and study end points, with the aim of optimizing the development of such combinations.

${ }^{1}$ ATIP-Avenir Group, UMR981, INSERM (French National Institute of Health and Medical Research), Gustave Roussy Cancer Campus, Villejuif, France. ${ }^{2}$ Nuclear Dynamics Unit UMR3664, National Centre for Scientific Research, Institut Curie, Paris, France. ${ }^{3}$ University Hospital Brussels, Brussels, Belgium.

${ }^{4}$ Drug Development Department (DITEP), Gustave Roussy Cancer Campus, Paris-Saclay University, Villejuif, France.

${ }^{5}$ These authors contributed equally: Daphné Morel, Daniel Jeffery, Geneviève Almouzni and Sophie Postel-Vinay

*e-mail: genevieve.almouzni@ curie.fr; sophie.postel-vinay@ gustaveroussy.fr

https://doi.org/10.1038 s41571-019-0267-4
Over the past decade, remarkable advances in anticancer therapy have been made, owing notably to the development of precision medicine and immunotherapy approaches as well as continuous improvements in the available therapeutic options and strategies. Nevertheless, the persistent ability of cancers to resist treatment remains a major challenge that limits the effectiveness all anticancer therapies.

Epigenetic dysregulation has major roles in tumour development, progression and acquisition of therapeutic resistance, in both solid and haematological malignancies $^{1}$. To date, however, the efficacy of 'epi-drugs' - drugs that target enzymes involved in epigenetic regulation of genome function (FIG. 1) - has mostly been confined to haematological cancers ${ }^{2}$, perhaps in part because solid tumours tend to arise from more-differentiated or even terminally differentiated cells with a reduced capability for epigenetic reprogramming. Furthermore, biomarkers for patient selection have crucially been lacking in early phase trials of epi-drugs, which have largely been conducted according to the historical 'one size fits all' approach. This strategy probably hampered the development of first-generation and second-generation epi-drugs, which are almost exclusively DNA methyltransferase (DNMT) inhibitors or histone deacetylase (HDAC) inhibitors (BOX 1). However, success with certain third-generation epi-drugs used according to precision medicine paradigms has provided new hope in the field of epigenetic therapy. For example, the isocitrate dehydrogenase 1 (IDH1) inhibitor ivosidenib received FDA approval on the basis of the results of a phase I trial involving patients with IDH1-mutant acute myeloid leukaemia (AML), only 4 years after the first patient was enrolled ${ }^{3}$. In addition, tazemetostat, an inhibitor of the histone-lysine $N$-methyltransferase enhancer of zeste homologue 2 (EZH2), has demonstrated efficacy in patients with EZH2-mutant haematological malignancies or highly aggressive solid tumours harbouring genetic aberrations affecting the SWI/SNF chromatin remodelling complex, such as SMARCB1 (also known as INI1) or SMARCA4 mutations or deletions ${ }^{4}$. Furthermore, responses to bromodomain and extra-terminal domain (BET) inhibitors, which target certain bromodomain-containing protein (BRD) family members, have been observed in patients with BRD4-rearranged NUT midline carcinoma ${ }^{5}$. 


\section{Key points \\ - The first generation of drugs targeting the epigenome (epi-drugs) were developed using a 'one size fits all' approach and proved to have disappointing efficacy in patients with solid tumours. \\ - The potential of epi-drugs to modulate the sensitivity of tumours to other anticancer drugs and to overcome therapy resistance presents major new avenues of clinical investigation. \\ - A new generation of epi-drugs, which were developed to be more specific for their targets and have promising activity in certain biomarker-selected populations, is now entering early phase clinical trials and are showing promising efficacy. \\ - Epi-drug development should follow a precision-medicine approach, with further identification of robust predictive biomarkers for patient selection and subsequent implementation of this strategy in clinical trials. \\ - Sequential treatment schedules and/or dosing at less than the maximum tolerated dose might improve the efficacy and tolerability of epi-drugs when used in combination with various other anticancer therapies.}

Cancers continuously evolve, in part through dynamic and reversible epigenetic changes that confer a fitness advantage. This epigenetic plasticity - a term used hereinafter in reference to reversible changes in epigenetic marks on DNA, histone and non-histone proteins, as well as the functional consequences of these alterations - is also involved in primary and acquired resistance to various anticancer therapies, through modulation of tumour cells or their microenvironment ${ }^{6,7}$ (Supplementary Figure 1). Epi-drugs might therefore be most effective when used in combination with other treatments and present particularly attractive strategies for sensitizing cancer cells to a given therapy and for overcoming acquired resistance mechanisms in a dynamic fashion. Of note, cancer stem cells, which are characterized by their abilities to self-renew and initiate cancer ${ }^{8}$, probably also have important roles in therapeutic resistance. The inherent phenotypic plasticity of these stem-like cells is key to this resistance and is regulated by both transcription factors and epigenetic changes. Indeed, some third-generation epi-drugs, such as lysine-specific histone demethylase 1A (LSD1) inhibitors, have been shown to disrupt the molecular changes underlying this plasticity. However, this topic has been reviewed elsewhere ${ }^{9}$ and will not be discussed further herein.

Despite robust preclinical evidence supporting epi-drug-containing combination therapies, clinical success with first-generation and second-generation epi-drugs has been limited. With the new generation of novel, selective epi-drugs currently entering clinical testing, learning from past successes and failures is crucial to maximizing future patient benefits. Herein, we provide a critical review of preclinical and clinical data supporting the use of epi-drugs in combination with other anticancer therapies in the treatment of patients with solid tumours. We also highlight the high stakes and challenges of successful epi-drug development and propose clinically relevant approaches to optimize this process.

\section{An appraisal of epi-drug combinations}

Epigenetic regulation influences all of the recognized hallmarks of cancer, which can each be targeted using various therapeutic approaches. These hallmarks comprise ten biological capabilities acquired during oncogenesis that drive and/or enable the development of cancer, as defined by Hanahan and Weinberg ${ }^{10}$. Various epi-drugs are able to enhance the effectiveness of four major pillars of the anticancer treatment armamentarium, namely genotoxic and/or cytotoxic treatments (encompassing chemotherapy and radiotherapy), hormone therapy, molecularly targeted therapy and immunotherapy ${ }^{11}$ (FIG. 2). In the following sections of this Review, we describe the synergistic mechanisms of epi-drug combinations stratified according to each of these four pillars of therapy, first discussing the preclinical evidence, followed by examples of corresponding clinical trials. The examples provided were selected to illustrate the specific challenges to the development of epi-drug combinations (including those relating to patient selection, treatment schedules, toxicities and efficacy assessments) and to highlight the most recent and most prominent clinical successes and failures. We also provide comprehensive lists summarizing past and ongoing trials of epi-drugs in combination with other anticancer therapies (Supplementary Tables 1 and 2 , respectively).

\section{Combinations with cytotoxic agents}

Chemotherapies. In preclinical studies assessing the effects of epi-drugs in combination with chemotherapy, synergy has mostly been achieved with DNA-damaging agents. HDAC inhibitors and DNMT inhibitors promote global chromatin relaxation, which facilitates DNA damage by genotoxic agents and interferes with DNA-damage repair (DDR) ${ }^{12}$ (FIG. 3a). HDAC inhibitors further impede DDR by disrupting ATM signalling, modulating the post-translational modification of non-histone substrates of HDACs, including p53 and $\mathrm{X}$-ray repair cross-complementing protein 6 (also known as Ku70), and by interfering with the regulation of histone marks that regulate $\mathrm{DDR}^{13}$. In addition, combined use of HDAC inhibitors and DNMT inhibitors at low doses might revert resistance to cytotoxic agents, in part, by inducing removal of acquired epigenetic alterations that drive the resistance phenotype ${ }^{14,15}$. Furthermore, epigenetic silencing of genes involved in shaping the tumour microenvironment (such as TGFBI) has been associated with resistance to taxanes and can be reversed with DNMT inhibitors, such as 5-azacytidine ${ }^{16}$, whereas defects in genes encoding components of chromatin remodelling complexes (such as SMARCA4) are correlated with sensitivity to taxanes and cisplatin ${ }^{17,18}$. Interestingly, Fillmore et al. ${ }^{19}$ showed that inhibition of EZH2 increases the sensitivity of SMARCA4-mutant or EGFR-mutant non-small-cell lung cancers (NSCLCs) to DNA topoisomerase 2 (TOP2) inhibitors, but conversely promotes resistance in tumours that are wild type for both genes. These findings highlight the importance of considering genomic characteristics of the tumour when selecting patients for therapy with particular epidrugs. Other third-generation epi-drugs, such as BET inhibitors and protein arginine $\mathrm{N}$-methyltransferase 5 (PRMT5) inhibitors, also interfere with DDR processes, raising the possibility of synergy with cytotoxic therapy (reviewed in detail elsewhere ${ }^{20,21}$ ). 


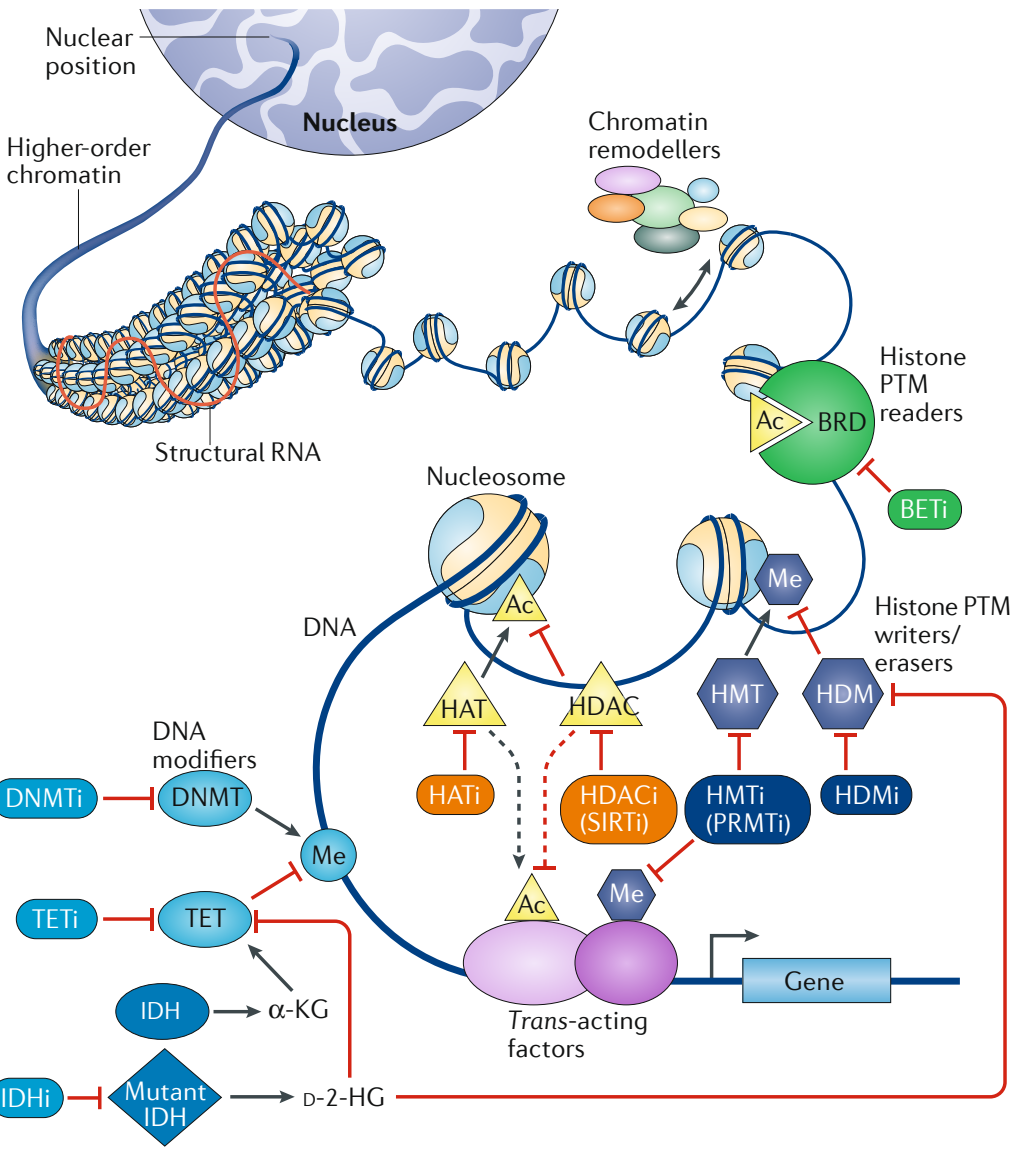

Fig. 1 | Targeting the epigenome. Genome function is regulated at multiple levels by $>800$ epigenetic enzymes. A predominant aspect of this regulation involves variations in the organization of genomic DNA within chromatin structures, the basic unit of which is the nucleosome core particle consisting of $146 \mathrm{bp}$ of DNA wrapped around a histone octamer. Epigenetic modifiers acting at this level comprise four nonexclusive categories: the 'writers', which add specific marks to DNA or the core histones H2A, H2B, H3, and H4; 'readers', which recognize these marks; 'erasers', which remove them; and the 'shapers', which remodel chromatin, mobilize nucleosomes or mediate histone exchange. Current epigenetic drugs (epi-drugs) target proteins belonging to the first three categories. These epi-drugs include inhibitors of DNA-modifying enzymes, such as DNA methyltransferases (DNMTs) that can methylate certain cytosine or adenosine nucleotides of DNA and ten-eleven translocation (TET) enzymes that catalyse the oxidation of 5-methylcytosine in DNA to initiate the process of active DNA demethylation. Another category of epi-drugs with effects on DNA methylation target gain-of-function mutant forms of isocitrate dehydrogenase (IDH) enzymes, which produce the oncometabolite D-2-hydroxyglutarate (D-2-HG) that competitively inhibits TET hydroxylases and thereby results in aberrant DNA methylation. By contrast, wild-type IDH enzymes support DNA demethylation by producing $\alpha$-ketoglutarate $(\alpha-K G)$, which is a cofactor for TET proteins. Other epi-drugs inhibit writers or erasers of histone arginine and/or lysine post-translational modifications (PTMs), including histone acetyltransferases (HATs), histone deacetylases (HDACs), histone methyltransferases (HMTs) and other protein arginine methyltransferases (PRMTs) and histone demethylases (HDMs), which can also act on non-chromatin factors. An additional class of epi-drugs inhibits readers of histone PTMs, such as bromodomain and extra-terminal domain (BET) family proteins (BRDs), which bind acetylated lysine residues of histones (as well as other non-chromatin factors). Epi-drugs targeting chromatin remodelling factors and histone chaperones are also under development, but are beyond the scope of this manuscript. SIRTi, sirtuin inhibitor. Figure adapted from REF. ${ }^{161}$, Springer Nature Limited. phase II studies ${ }^{24,25}$ conducted to evaluate this strategy by combining decitabine with carboplatin in the treatment of women with recurrent and/or progressing ovarian cancer illustrate certain challenges associated with epi-drug-chemotherapy combinations. In one of these trials ${ }^{25}$, low-dose decitabine $\left(10 \mathrm{mg} / \mathrm{m}^{2}\right)$ was administered on days $1-5$ of a 28 -day cycle, which seemed to result in better tolerability than high-dose decitabine ( 45 or $90 \mathrm{mg} / \mathrm{m}^{2}$ ) administered on day 1 of each cycle in the other trial $^{24}$ (Supplementary Table 1). Differences in the trial designs and patient populations precludes any formal comparison of these trials, although the level of efficacy - in terms of objective response rate (ORR) and progression-free survival (PFS) - in the trial of low-dose decitabine (which included patients with platinum-refractory disease) also seemed to be superior to that observed in the trial of the high-dose regimen (in which patients with partially platinum-sensitive disease were enrolled), thus supporting the selection of the low-dose regimen for further testing in a subsequent phase III trial (Supplementary Table 2). Interestingly, both studies showed a high incidence of carboplatin-related hypersensitivity reactions with the combination (35\% and $67 \%$ ), potentially resulting from decitabine-induced demethylation of genes encoding cytokines involved in commitment of naive $\mathrm{T}$ cells to the Thelper $1\left(\mathrm{~T}_{\mathrm{H}} 1\right)$ and T helper $2\left(\mathrm{~T}_{\mathrm{H}} 2\right)$ lineages and IgE-mediated hypersensitivity reactions ${ }^{24,26}$. Global decitabine-induced 5-methylcytosine demethylation of DNA from peripheral blood mononuclear cells (PBMCs) was also noted in both trials $^{24,25}$. This effect was dose-dependent ${ }^{27}$, which is in contrast to the findings of other clinical trials indicating that the pharmacodynamic modulation induced by certain epi-drugs reaches a plateau at doses well below the maximum tolerated dose $\mathrm{e}^{4,28}$. Notably, Matei et al. ${ }^{25}$ identified that patients with a PFS duration of $>6$ months have higher numbers of demethylated genes in tumour biopsy samples and in PBMCs, suggesting that PBMC DNA methylation status could provide information on tumour DNA methylation and serve as a pharmacodynamic or predictive biomarker.

Overall, despite the preclinical data showing that HDAC inhibitors and DNMT inhibitors potentiate the activity of cytotoxic chemotherapy, clinical results have been disappointing: three out of the five key randomized trials of such combinations were halted owing to a lack of efficacy and/or unfavourable toxicity profiles when compared with chemotherapy alone. Such undesired systemic toxicities and limited efficacy might warrant investigation of targeted delivery of epi-drugs, for example, as payloads of antibody-drug conjugates (ADCs) or liposomal particles. Moreover, most - if not all - trials of epi-drug-chemotherapy combinations have been performed in unselected patient populations (that is, without the use of predictive molecular biomarkers). Thus, appropriate patient selection using rational epigenetic biomarkers might produce clinically meaningful improvements in the therapeutic window and new therapeutic opportunities. For example, evaluating SLFN11 or PCFT promoter hypermethylation using clinical grade assays could potentially enable the identification of patients who would benefit most from 


\section{Box 1 | Generations of epi-drugs}

Multiple generations of epigenetic drugs (epi-drugs) have been developed. First-generation and second-generation epi-drugs include DNA methyltransferase inhibitors (DNMTi) and histone deacetylase inhibitors (HDACi), some of which have already been approved for the treatment of certain haematological malignancies ${ }^{2}$. DNMTi are pyrimidine analogues that are incorporated into DNA during replication and form covalent DNMT-drug adducts that trigger the activation of DNA damage response and can, ultimately, lead to apoptosis, thus explaining the dual epigenetic and cytotoxic effects of these drugs. With the exception of sirtuin inhibitors, which inhibit nicotinamide adenine dinucleotide ( $\mathrm{NAD}^{+}$)-dependent class III histone deacetylases, $\mathrm{HDACi}$ inhibit the $\mathrm{Zn}^{2+}$-dependent catalytic activity of either all (pan-HDACi) or single HDAC enzymes (for example, HDAC6i). First-generation DNMTi (azacytidine and decitabine) and HDACi (vorinostat and romidepsin) have unfavourable pharmacokinetic characteristics and target selectivity, which led to the development of second-generation DNMTi (such as zebularine and guadecitabine) and $\mathrm{HDACi}$, (including the hydroxamic acid $\mathrm{HDACi}$ belinostat and panobinostat, the benzamide HDACi tucidinostat (also known as chidamide) and carboxylic acid derivative $\mathrm{HDACi}$ (such as valproic acid)), with improved chemical properties under physiological conditions.

Third-generation epi-drugs include, among others, bromodomain and extra-terminal domain inhibitors (BETi), histone methyltransferase inhibitors (HMTi) and histone demethylase inhibitors (HDMi) and protein arginine methyltransferase inhibitors (PRMTi). BETi disrupt the recognition of acetylated lysine residues on histones, a mark associated with active transcription, by BET-containing reader proteins, including certain members of the bromodomain-containing protein (BRD) family. HMTi (including enhancer of zeste homologue 2 inhibitors and DOT1-like histone-lysine methyltransferase inhibitors), HDMi (such as lysine-specific histone demethylase 1A inhibitors) or PRMTi (for example, targeting PRMT1 or PRMT5) reduce the catalytic activity of the specific target enzyme or, in the specific case of embryonic ectoderm development (EED) protein inhibitors, destabilize the enzymatic complex containing the target protein.

the use of DNMT inhibitors to reverse resistance to platinum-based agents ${ }^{29}$ or pemetrexed ${ }^{30}$, respectively.

Radiotherapy. In preclinical studies, HDAC, DNMT, $\mathrm{EZH} 2$ or BET inhibition potentiates the antitumour activity of radiotherapy by disrupting DDR and the cell cycle and increasing oxidative stress ${ }^{31-35}$ (FIG. 3a). Transient HDAC inhibition with valproic acid (before and up to 24 hours after irradiation) results in radiosensitization of human glioma cell lines - a schedule that might limit the systemic toxicities associated with chronic HDAC inhibition ${ }^{36}$. Interestingly, BET inhibition can decrease the risk of radiation-induced lung fibrosis and has a radioprotective effect on irradiated non-malignant lung tissue in mouse models, but conversely radiosensitizes thoracic cancer cells (that is, NSCLC, breast or oesophageal cancer cells) in vitro ${ }^{37}$. This potential ability to differentially modulate radiosensitivity according to cell lineage, if recapitulated in humans, could provide new therapeutic opportunities.

Certain DNMT inhibitors, such as the cytidine analogues decitabine and 5-azacytidine, can be incorporated into DNA and/or RNA; thus, these agents are particularly strong radiosensitizers in all tissues and the combination of these epi-drugs with radiotherapy is not recommended owing to concerns regarding toxicity. In clinical trials, combining HDAC inhibitors with radiotherapy, usually concurrently and sometimes in addition to chemotherapy, has mostly resulted in increased levels of toxicity and minimal patient benefit (Supplementary Table 1). Two phase I studies, however, have provided encouraging results. First, the combination of the HDAC inhibitor vorinostat with capecitabine and radiotherapy was well tolerated in 21 patients with localized pancreatic ductal adenocarcinoma and led to four R0 resections among 11 patients who underwent surgical exploration and a median overall survival (OS) duration of 1.1 years for the entire cohort, which according to the investigators compared favourably with the results of other phase I studies of chemoradiotherapy in such a patient population ${ }^{38}$. Second, the combination of vorinostat and vectorized internal radiotherapy with ${ }^{131}$ I-metaiodobenzylguanidine in children with relapsed and/or refractory high-risk neuroblastoma led to an ORR of $12 \%$ at all dose levels and $17 \%$ at the recommended phase II dose; ${ }^{39}$ this regimen is now being evaluated in a randomized phase II trial (Supplementary Table 2). Why these two studies had promising results whereas other clinical trials of epi-drug-radiotherapy combinations did not is unclear, although the apparently greater permissivity of tumours to epigenetic reprogramming in the latter study might be explained by the paediatric nature of the disease (which is likely to have arisen during early development, when epigenetic processes have key roles in determining cell fate). These results call for the implementation of biomarkers for patient selection. For example, a vorinostat sensitivity transcriptional signature (sig-139), which is enriched for the expression of genes involved in DNA synthesis, repair and chromatin remodelling as well as RNA synthesis, splicing and processing has been identified using a panel of cell lines, with positive associations with PFS and OS observed in patients with newly diagnosed glioblastoma treated with vorinostat plus standard temozolomide and radiation therapy ${ }^{40}$.

\section{Combinations with hormone therapy}

Oestrogen receptor-positive breast cancer. Oestrogen receptor- $\alpha(E R \alpha)$, the pivotal oncogenic driver of $70 \%$ of breast cancers, is released from heat shock protein 90 (HSP90) upon binding with its endogenous ligands, predominantly oestradiol. The ER $\alpha$-oestrogen complex subsequently translocates into the nucleus and binds to oestrogen-responsive elements - a process tightly regulated by chromatin-remodelling factors ${ }^{41,42}$. Preclinically, HDAC inhibitors have been shown to increase the antitumour efficacy of, or reverse resistance to, hormone therapies in models of ERa-positive breast cancer ${ }^{43-45}$. HDAC inhibition interferes with ERa signalling pathway at multiple levels, including transcriptional suppression of ESR1 (which encodes ER $\alpha$ ), reductions in ER $\alpha$ mRNA and protein stability and modulation of the transcription of ERa targets as well as other genes involved in cell proliferation and metastasis ${ }^{46,47}$ (FIG. 3b). HDAC6 inhibition, specifically, leads to HSP90 hyperacetylation, which disrupts the HSP90-ERa association and favours ERa ubiquitination and proteasomal degradation ${ }^{48}$. Selective HDAC6 inhibitors are, therefore, interesting candidates for future clinical evaluation ${ }^{48,49}$.

BET inhibition also suppresses the growth of tamoxifen-resistant tumour cells and xenografts in combination with the anti-oestrogen fulvestrant ${ }^{50,51}$; the underlying mechanisms include inhibition of the BRD3 and/or BRD4-dependent recruitment of the histonelysine $N$-methyltransferase NSD2 (also known as WHSC1) 


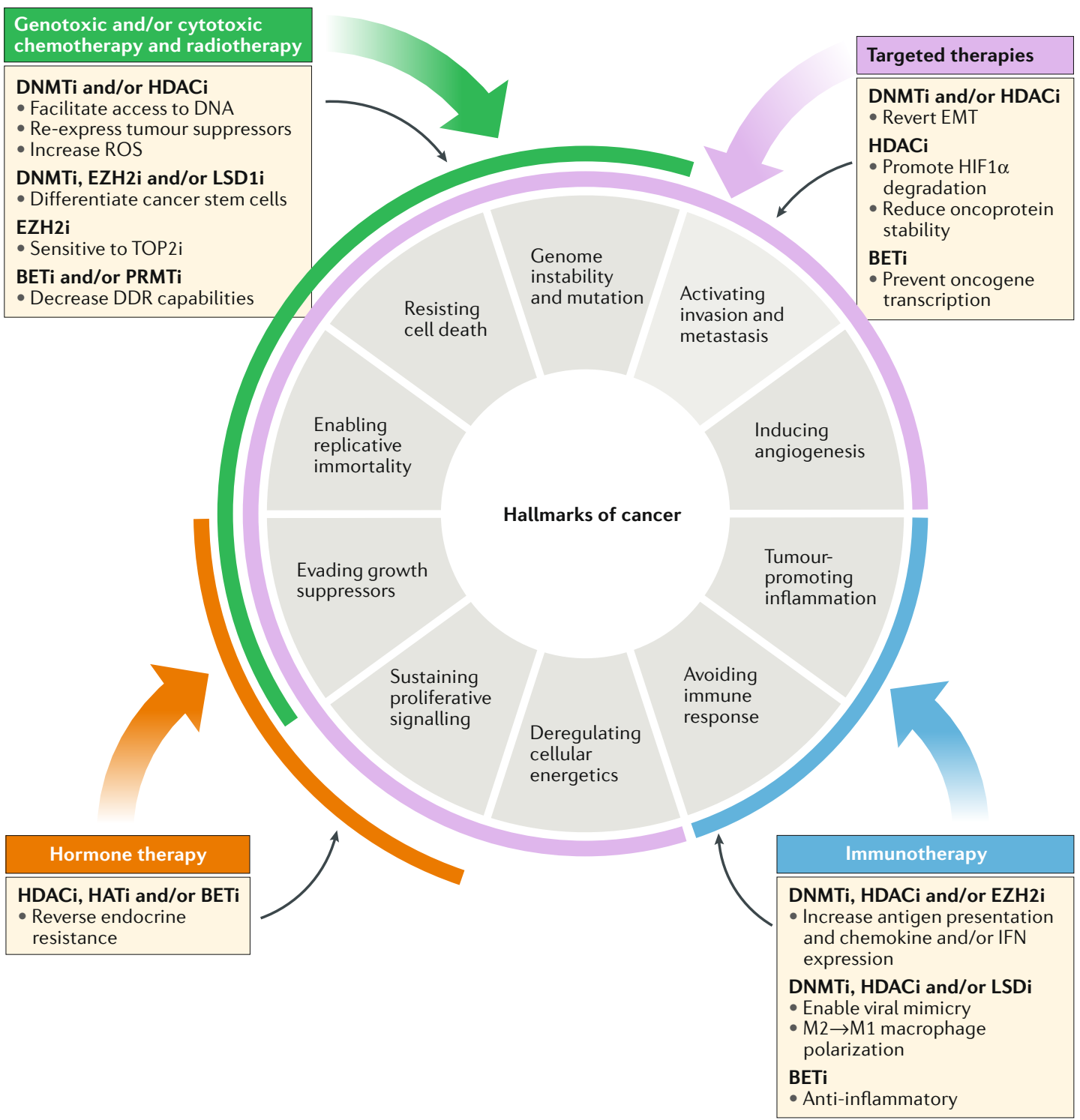

Fig. 2 | Epi-drugs can enhance the activity of other anticancer therapies to counteract the hallmarks of cancer. Current prominent types of anticancer therapeutics have counteracting effects against various hallmarks of cancer (defined by Hanahan and Weinberg ${ }^{10}$ in 2011), as indicated by the coloured arcs surrounding the hallmarks of cancer wheel. Cytotoxic and/or genotoxic chemotherapies and radiotherapy (green arc), hormone therapy (orange arc), immunotherapy (blue arc) and targeted therapies (purple arc) each counteract specific hallmarks, with some overlap. Epigenetic drugs (epi-drugs) can enhance the activity of these anticancer therapies through various mechanisms; examples of the relevant categories of epi-drugs and their augmenting effects observed in preclinical studies are indicated in the figure for each type of therapy. Accordingly, the combination of epi-drugs with other anticancer therapies can lead to sensitization to treatment and/or reversal of resistance. BETi, bromodomain and extra-terminal domain inhibitor; DDR, DNA damage repair; DNMTi, DNA methyltransferase inhibitor; EZH2i, enhancer of zeste homologue 2 inhibitor; EMT, epithelial-to-mesenchymal transition; $\mathrm{HATi}$, histone acetyltransferase inhibitor; $\mathrm{HDACi}$, histone deacetylase inhibitor; $\mathrm{HIF1} 1 \alpha$, hypoxia-inducible factor 1 $\alpha$; IFN, interferon; LSD1i, lysine-specific demethylase 1 inhibitor; PRMTi, protein arginine methyltransferase inhibitor; ROS, reactive oxygen species; TOP2i, DNA topoisomerase 2 inhibitor. Adapted with permission from REF. ${ }^{10}$, Elsevier.

which monomethylates and/or dimethylates histone $\mathrm{H} 3$ lysine 36 to generate $\mathrm{H} 3 \mathrm{~K} 36 \mathrm{me} 1$ and $\mathrm{H} 3 \mathrm{~K} 36 \mathrm{me} 2$ marks that are commonly associated with active transcription, and disruption of elongation-associated phosphorylation events on RNA polymerase II that results in stalled transcription, thus ultimately reducing the expression of ESR1 and ERa target genes ${ }^{50-53}$. Similarly, single-agent activity and synergism with anti-oestrogen therapies has been demonstrated in preclinical studies of CPI-1, a selective inhibitor of the CREB-binding protein (CBP) and histone acetyltransferase p300 (EP300) transcriptional co-activator proteins, via a mechanism involving disruption of the core ERa signalling complex ${ }^{54}$.

The results of two phase II studies demonstrate that combinations of HDAC inhibitors and an antioestrogen or aromatase inhibitor have acceptable tolerability, with promising efficacy (Supplementary Table 1). The first trial, a single-arm study of vorinostat 

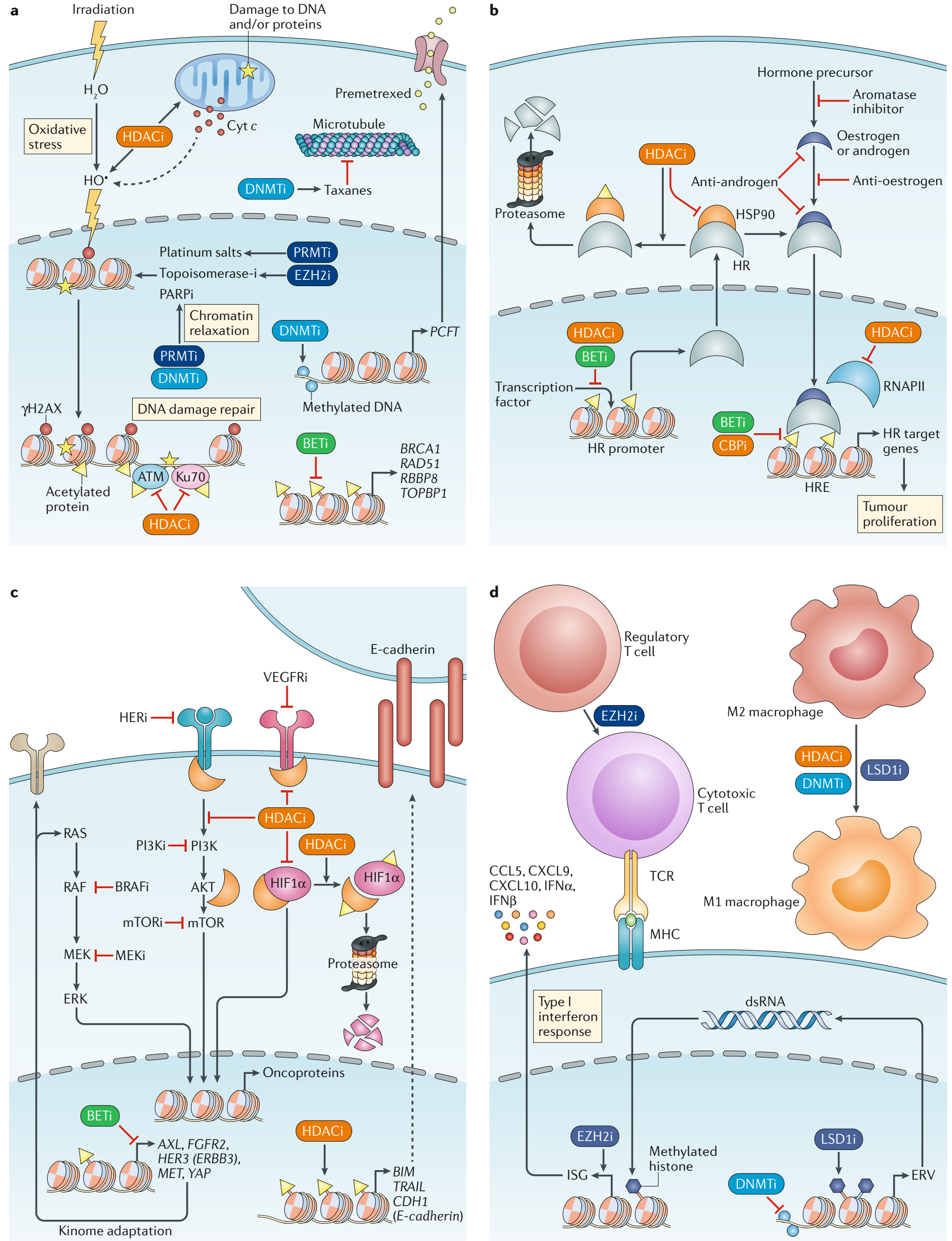
4 Fig. 3 | Rationale for epi-drug combination, with examples of synergy or reversal of resistance mechanisms. Overview of epigenetic drug (epi-drug)-induced modulation of sensitivity to four pillars of anticancer therapy: radiotherapy, cytotoxic chemotherapy and DNA damage repair-targeting therapies (part a); hormonotherapy (part b); targeted therapies (part c); and immunotherapies (part d). Prominent processes are detailed here but others exist. Histone deacetylase inhibitors (HDACi) and DNA methyltransferase inhibitors (DNMTi) synergise with genotoxic and/or cytotoxic therapies by interfering with the DNA damage response (DDR), notably through increased DNA damage and disruption of the function of DDR proteins. Bromodomain and extra-terminal domain inhibitors (BETi) globally decrease DDR protein levels, resulting in functional DDR defects. Protein arginine methyltransferase 5 inhibitors (PRMTi), among other mechanisms, more specifically induce aberrant splicing of DNA repair factors, thereby impairing their activity (part a). HDACi interfere with hormone receptors (HR) at multiple levels, including transcriptional regulation, mRNA and protein stability (notably through modulation of acetylation levels of their chaperone protein HSP90) and transcription of nuclear target genes. BETi suppress the transcription of the HR themselves or their target genes (part b). HDACi modulate the stability of several tyrosine kinase receptors and downstream proteins, notably through modulation of acetylation levels of HSP90. HDAC4/6 inhibitors more specifically reduce hypoxia-inducible factor $1 \alpha(\mathrm{HIF} 1 \alpha)$ stability and activity, thereby synergizing with anti-angiogenic agents. BET inhibitors prevent the transcription of alternative tyrosine kinase receptors and proteins that drive resistance through the 'kinome adaptation' mechanism (part c). Treatment of cancer cells with enhancer of zeste homologue 2 inhibitors (EZH2i), lysine-specific histone demethylase $1 \mathrm{~A}$ inhibitors (LSD1i), or DNMTi favours chemokine-dependent T cell attraction following enhanced expression of CC-chemokine ligand 5 (CCL5), CXC-chemokine ligand 9 (CXCL9), CXCL10 or CXCL12. LSD1i and DNMTi also stimulate type I interferon response through increased expression of endogenous retroviral elements (ERV), which eventually triggers T cell-mediated antitumour immunity following double-strand RNA (dsRNA) stress. Epi-drugs can also modulate immune cell transcriptional programmes. EZH2i elicit functional alterations in regulatory $T$ cells and enhance the cytotoxicity of effector T cells, while other epi-drugs, such as LSD1i, DNMTi and HDACi, decrease myeloid-derived suppressor cell activity and favour a M1-like macrophage-mediated antitumour immune response (part d). ATM, ataxia telangiectasia mutated; BRAFi, BRAF inhibitor; $\mathrm{CBPi}$, CREB-binding protein inhibitor; Cyt c, cytochrome c; HERi, HER (ErbB) family receptor tyrosine kinase inhibitor; HRE, hormone response element; ISG, interferon-stimulated genes; MEKi, MEK inhibitor; mTORi, mTOR inhibitor; PARPi, poly(ADP-ribose) polymerase inhibitor; PI3Ki, PI3K inhibitor; RNAPII, RNA polymerase II; TCR, T cell receptor; VEGFRi, VEGFR inhibitor.

combined with tamoxifen in 43 patients with ER-positive, endocrine-resistant metastatic breast cancer ${ }^{55}$, revealed durable (lasting $>24$ weeks) objective responses in $19 \%$ and disease stabilization in $21 \%$ of patients. In the second trial, ENCORE 301, a randomized placebo-controlled study of entinostat added to exemestane in 130 women with ER-positive, endocrine-resistant advanced-stage breast cancer ${ }^{56}$, the entinostat combination resulted in numerical but not statistically significant improvements in PFS (median 4.3 months compared with 2.3 months in the placebo group; $P=0.11$ ) but a striking significant improvement in the exploratory OS end-point (median 28.1 months versus 19.8 months; $P=0.036$ ). In particular, patients with a percentage change in the level of PBMC pan-protein lysine acetylation above the study median had a better median PFS than those with a percentage change below the median (8.6 months versus 2.8 months; HR 0.32 , 95\% CI 0.13-0.79). Immunological analyses revealed significant depletion of blood granulocytic and monocytic myeloid-derived suppressor cells (MDSCs; $P=0.029$ and $P=0.002$, respectively), as well as decreased CD40 expression in immature and monocytic MDSCs $(P=0.007$ and $P=0.011$, respectively) and increased HLA-DR expression in $\mathrm{CD} 14^{+}$monocytes and enrichment of this CD $14^{+} \mathrm{HLA}-\mathrm{DR}{ }^{\text {hi }}$ monocyte subset $(P=0.015 \text { and } P=0.0004 \text {, respectively })^{57}$. Whether the 8.3-month OS benefit observed in ENCORE 301 resulted from beneficial effects on hormone resistance, cancer stem cells, cell differentiation, anticancer immunity and/or sensitization to subsequent therapies is unknown. Regardless, this finding led to the designation of entinostat plus exemestane as a breakthrough therapy by the FDA in postmenopausal women with advanced-stage hormone receptor-positive, endocrineresistant breast cancer, and supported the development of the E2112 study ${ }^{58}$. Results of this randomized, double-blind, placebo-controlled phase III trial, in which OS and PFS are co-primary end-points, are expected in 2021 and should shed light on the mechanisms underlying the benefits of adding entinostat to exemestane (if confirmed) and on relevant biomarkers for such combinations. Importantly and further supporting the above strategy, the randomized, double-blind, placebo-controlled, phase III ACE study, in which the addition of the HDAC inhibitor tucidinostat to exemestane was evaluated in postmenopausal patients with advanced-stage hormone receptor-positive breast cancer that had progressed after previous endocrine therapy, revealed a significant prolongation of PFS in the experimental arm (7.5 months versus 3.8 months; HR 0.74, 95\% CI 0.58-0.98; $P=0.03)^{59}$.

Hormone-sensitive prostate cancer. Observations similar to those described in preclinical models of ERa-positive breast cancer have been reported in models of both hormone-sensitive and castration-resistant prostate cancer models (CRPC) ${ }^{60-63}$ (FIG. 3b). Additionally, BET inhibition prevents binding of the androgen receptor (AR) to its target binding sites in the genome and the subsequent recruitment of RNA polymerase II; accordingly, addition of the BET inhibitor JQ1 to the anti-androgen enzalutamide or of the BET inhibitor OTX-015 to the AR-agonist ARN-509 markedly reduced the growth of enzalutamide-resistant prostate cancer xenografts in mice ${ }^{64,65}$. Early phase clinical trials of BET inhibitors combined with next-generation anti-androgens in patients with CRPC are ongoing (Supplementary Table 2).

In a phase II trial in patients with CRPC resistant to second-line antiandrogen therapy ${ }^{66}$, the addition of the HDAC inhibitor panobinostat to the second-generation anti-androgen bicalutamide increased the median PFS in comparison with that of historical control groups (34 weeks versus 16 weeks). However, this potential benefit was only observed with high-dose panobinostat ( $40 \mathrm{mg}$ triweekly), which was associated with an increase in the incidence of severe toxicities: treatment interruption and dose reductions were needed in $27.5 \%$ and $41 \%$ of patients, respectively, versus $11.5 \%$ and $4 \%$ of patients receiving $20 \mathrm{mg}$ triweekly. This finding suggests that more selective compounds with an improved therapeutic window might be required for the long-term clinical development of such combinations.

\section{Combinations with targeted therapy}

ErbB-targeted therapies. Preclinical evidence shows that resistance to agents targeting ErbB (also known as HER) family receptor tyrosine kinases (RTKs) can be epigenetically driven and thus reverted by epi-drugs (FIG. 3c). 
Examples include the reversion of epithelial-tomesenchymal transition (EMT) that can occur with ErbB-targeted therapies through HDAC inhibition ${ }^{8}$ or the hyperacetylation of HSP90 chaperone proteins resulting from HDAC6 inhibition, which decreases interactions of HSP90 with stabilization of multiple RTKs, including EGFR (ErbB-1) ${ }^{67}$. Target-specific mechanisms also exist. A deletion polymorphism that results in alternative splicing of BCL2L11 (encoding the pro-apoptotic BCL-2-like protein 11, also known as BIM) is associated with resistance of EGFR-mutant NSCLC cell lines to EGFR tyrosine kinase inhibitors (TKIs) and shorter PFS durations in series of patients with EGFR-mutant NSCLC who received such TKIs (6.6 months compared with 11.9 months in those without the polymorphism $)^{68}$; notably, alternative splicing of BCL2L11 has been shown to be HDAC3dependent and can be reverted by vorinostat in preclinical models ${ }^{69}$. The clinical relevance of this approach is currently being evaluated in a phase I trial of vorinostat and gefitinib in patients with EGFR-mutant NSCLC harbouring the BCL $2 L 11$ deletion polymorphism (Supplementary Table 2).

Targeting BRD4 using BET inhibitors also overcomes resistance to ErbB-targeted agents in preclinical models of various tumour types, including anti-EGFR antibodies in head and neck squamous cell carcinoma (HNSCC) and the dual EGFR and HER2 (ErbB-2) TKI lapatinib in HER2-positive breast cancer, notably through the abrogation of the transcription of alternative kinases that drive acquired resistance, such as MET, AXL, FGFR2 or HER3 (ErbB-3) - a phenomenon referred as 'kinome adaptation' ${ }^{70-72}$ (FIG. 3 C). In in vitro models of lapatinib-resistant breast cancer, the combination of JQ1 and lapatinib has even been reported to be superior to lapatinib plus other TKIs targeting the bypass pathways, and removal of lapatinib from the media allowed the cells to regrow despite continued JQ1 monotherapy, suggesting that BET inhibition re-sensitized resistant cells rather than simply synergizing with lapatinib $^{70,71}$. RNA sequencing (RNA-seq) revealed that the effect of BET inhibition was remarkably specific, as JQ1 monotherapy resulted in the downregulation of only $8 \%$ of all expressed genes, but suppressed $27 \%$ of lapatinib-induced genes when used in combination with lapatinib ${ }^{70,71}$. Importantly, in HNSCC cell lines with acquired resistance to the anti-EGFR antibody cetuximab, this heterogeneous kinome adaptation is associated with increased levels of BRD4 expression ${ }^{72}$, which might therefore serve as patient selection biomarker for this combination.

Multiple phase I and/or II studies have shown the feasibility of combining HDAC inhibitors and ErbB inhibitors (predominantly EGFR TKIs in patients with NSCLC, but also the anti-HER2 antibody trastuzumab in patients with breast cancer) although no clear signs of delays or reversal of resistance have been observed (Supplementary Table 1), perhaps because patients with epigenetically driven resistance to trastuzumab were not selected. For example, patients with NSCLC enrolled in the ENCORE-401 randomized phase II study of erlotinib plus either entinostat or placebo were not selected on the basis of EGFR mutational status or using epigenetic biomarkers $^{73}$. Likewise, in the phase I/II IVORI study involving unselected patients with NSCLC ${ }^{74}$, gefinitib plus vorinostat resulted in a median PFS of 9.1 months that did not seem to be better than historical data ${ }^{75}$. In this regard, an ongoing randomized, placebo-controlled, double-blind phase II/III trial (NCT02416739) of gefitinib or erlotinib with or without the HDAC3 inhibitor nicotinamide in patients with EGFR-mutant NSCLC should provide more relevant data and could potentially enable the identification of patient selection biomarkers for this combination (Supplementary Table 2).

Anti-angiogenic agents. Tumour angiogenesis is mainly controlled by hypoxia-inducible factor $1 \alpha$ (HIF1 $\alpha)$ and its transcriptional target VEGF. Inhibition of HDAC4 and/or HDAC6 reduces HIF1a stability and transcriptional activity by promoting its acetylation, polyubiquitination and subsequent proteasome degradation ${ }^{76}$. Accordingly, HDAC inhibitors and VEGF inhibitors have synergistic anti-angiogenic and antitumour effects in mouse models of various cancers ${ }^{77,78}$.

Multiple early stage clinical trials have been conducted to evaluate the safety of combinations of HDAC inhibitors and anti-angiogenic agents, including vorinostat with bevacizumab for clear cell renal cell carcinoma $(\mathrm{RCC})^{79}$, resminostat with sorafenib for hepatocellular carcinoma ${ }^{80}$ and panobinostat with bevacizumab for high-grade glioma ${ }^{81,82}$ (Supplementary Table 1). All of these trials provide evidence that the combinations are tolerable and provided evidence of clinical benefit ${ }^{79-82}$. The most recently reported phase I study was conducted to assess the combination of abexinostat and pazopanib, with durable responses in seven of ten patients with pazopanib-refractory solid tumours, including one response lasting $>3.5$ years ${ }^{83}$. Among the 22 patients with RCC (including ten and eight patients with prior progression on pazopanib and anti-angiogenic agents, respectively), the ORR was $27 \%$ and the median duration of response was 10.5 months. Notably, histone acetylation and HDAC2 expression in PBMCs were positively correlated with durable responses to treatment ${ }^{83}$. These promising results led to the initiation of the ongoing randomized, double-blind, placebo-controlled phase III RENAVIV trial of the same combination, with a crossover design, which will enable a thorough evaluation of clinical benefit in patients with VEGFR TKI-naive advanced-stage RCC and could provide opportunities for biomarker identification (Supplementary Table 2).

PI3K, AKT and/or mTOR inhibitors. Findings of several preclinical studies indicate the potential of combinations of HDAC inhibitors and PI3K-AKT-mTOR pathway inhibitors, including the observations that HDAC inhibition can destabilize $\mathrm{AKT}^{84,85}$, suppress survivin expression ${ }^{86}$, inhibit EMT and invasiveness, or increase oxidative stress $^{87,88}$, and induce autophagy ${ }^{89}$. Inhibition of HDACs and the PI3K-AKT-mTOR pathway also converges on the upregulation of tumour suppressor genes, such as FOXO1 in MYC-driven medulloblastoma ${ }^{90}$ (FIG. 3c). Synergy also occurs between mTOR 
inhibitors and BET inhibitors in preclinical models of triple-negative breast cancer ${ }^{91}$. Interestingly, BET inhibition can both prevent and revert resistance to mTOR inhibition in such models ${ }^{91}$. Similar to the aforementioned observations relating to countering the kinome adaptation mechanism of resistance, transcriptional upregulation of RTKs upon PI3K inhibition can be overcome by preventing BRD4 binding at the promoter regions of RTK genes using BET inhibitors ${ }^{92}$. Furthermore, when single-agent treatments are ineffective, dual PI3K-BET inhibition can induce apoptosis across multiple cancer cell lines and mammary tumour models ${ }^{92}$. Similar results have been reported for preclinical models of epithelial ovarian cancer and neuroblastoma, in which PI3K inhibition overcomes resistance to BET inhibitors ${ }^{93,94}$. These data pose a considerable challenge to the drug development dogma that drugs that do not display single-agent activity should not progress beyond phase I testing ${ }^{95}$ and suggests that certain drugs might only be effective when rationally used in combinations. This approach might be especially pertinent with PI3K inhibitors and first-generation epidrugs, the single-agent activity of which has thus far been limited in patients with solid tumours.

Phase I trials in which mTOR inhibitors were combined with vorinostat in patients with various advanced-stage solid tumours have provided some evidence of therapeutic activity, but thrombocytopenia limited doses to levels below the full dose of each agent individually, suggesting that synergistic effects also occur in non-malignant cells ${ }^{96,97}$ (Supplementary Table 1). Again, these findings warrant the evaluation of discontinuous and/or sequential drug administration schedules, targeted drug delivery and/or biomarker-driven patient selection in order to improve the therapeutic index. Early phase trials of HDAC inhibitors combined with mTOR inhibitors are ongoing in patients with various types of solid tumour, including the combination of everolimus and panobinostat in patients with H3.3 or H3.1 K27M-mutant glioma (NCT03632317; Supplementary Table 2).

$B R A F$ and/or MEK inhibitors. In preclinical studies, HDAC inhibitors revert acquired resistance to BRAF and/or MEK inhibitors, notably, by upregulating transcription of the caspase 8 inhibitor c-FLIPL ${ }^{98}$, modulating PI3K signalling ${ }^{99}$ or downregulating melanocyte lineage pathways (restoration of which drives adaptive drug resistance in melanoma cells) ${ }^{100}$ (FIG. 3c). In 2019, HDAC inhibition was further shown to dramatically enhance the effectiveness of BRAF and/or MEK inhibition in preclinical models of both sensitive (BRAF-mutant) and resistant (NRAS-mutant or NF1-mutant) RAS pathway-driven melanoma ${ }^{101}$. Specifically, HDAC inhibitors induced chemical synthetic lethality via suppression of the non-homologous end-joining DDR pathway on a background of BRAF and MEK inhibitor-mediated disruption of the alternative homologous recombination pathways ${ }^{101}$. Importantly, MGMT expression was found to be characteristic of tumours with inherent defects in DNA repair and to predict responsiveness to such combination therapy ${ }^{101}$.
BET inhibitors also synergize with BRAF inhibitors in preclinical models of $B R A F$-mutant melanoma, which leads to increased cytotoxicity and epigenetic modulation of dedifferentiated precursor cells ${ }^{102,103}$. Furthermore, BET inhibition can overcome resistance to dual MEK and TBK1 inhibition in KRAS-driven NSCLC models by blocking transcriptional activation of YAP1, which is a core component of the Hippo signalling pathway that often contributes to tumorigene$\mathrm{sis}^{104}$. Interestingly, the investigators who conducted this study anticipated that continuous BET inhibition would not be tolerable, and used in vitro pharmacodynamic modelling to optimize an intermittent sequential administration schedule that was tolerable and proved to be equally effective in mice. Clinical data from studies evaluating such combinations are not currently available, but considering that the toxicities have been a major challenge to the application of epi-drug combinations, with long-term toxicities often being a limiting factor ${ }^{96,105,106}$, such intermittent scheduling approaches should be encouraged and applied more systematically.

PARP inhibitors. Preclinical synergy between HDAC inhibitors and poly(ADP-ribose) polymerase (PARP) inhibitors has been reported in models of triple-negative breast $^{107}$, prostate ${ }^{108}$ and ovarian cancer ${ }^{109}$. Mechanistically, HDAC inhibitors induce a 'BRCAness' phenotype following downregulation of proteins involved in homologous recombination, including RAD51, BRCA1 and UHRF1. Notably, the combination of olaparib and vorinostat is to be evaluated in patients with metastatic breast cancer (NCT03742245). Furthermore, in the past 2 years, the results of four independent studies have shown a strong synergy between PARP inhibitors and BET inhibitors, notably, in preclinical models of homologous recombination-proficient ovarian cancer ${ }^{110-113}$ (FIG. 3a). Mechanistically, BET inhibitors downregulate various proteins involved in homologous recombination, including BRCA1, BRCA2, RAD51 and RBBP8 (also known as CtIP), suggesting broad effects on the expression DDR-related genes rather than a targeted synthetic lethality (as epitomized by PARP inhibition in cancers associated with germline BRCA mutations). Interestingly, a reciprocal observation was made in models of BET inhibitor-resistant CRPC, whereby acquired polycomb repressive complex 2 (PRC2)-mediated silencing of DDR-related genes led to increased sensitivity to PARP inhibitors ${ }^{114}$. Combinations of PARP and BET inhibitors have not yet been evaluated in clinical studies. We anticipate that rational patient selection and optimal dosing and scheduling will be key to maximizing the therapeutic window and, thus, potential clinical utility, considering that thrombocytopenia is a dose-limiting toxicity (DLT) of HDAC, PARP and BET inhibitors.

Epi-drug-epi-drug combinations. Owing to the interplay among various epigenetic processes, combining several epi-drugs might be a promising approach to anticancer therapy. For example, overexpression of KMT2A (which encodes lysine $N$-methyltransferase $2 \mathrm{~A}$, also known as MLL1) drives resistance of EZH2-aberrant cancers to $\mathrm{EZH} 2$ inhibition via the interaction of MLL1 with the 
CBP and EP300 co-activators, which results in losses of H3K27 methylation and reciprocal gains in $\mathrm{H} 3 \mathrm{~K} 27$ acetylation, thereby triggering oncogenic transcriptional reprogramming; however, BET inhibition disrupts signalling mediated by $\mathrm{H} 3 \mathrm{~K} 27$ acetylation and re-sensitizes cancer cells to EZH2 inhibitors ${ }^{115}$. Similarly, BET inhibition can reverse resistance to DNMT inhibitors in preclinical models of colorectal cancer $(\mathrm{CRC})^{116}$.

\section{Combinations with immunotherapies}

Antitumour immunity involves the complex interplay among immune, cancer and stromal cells. Specific DNA-modifying or histone-modifying enzymes and histone chaperones, including DNMTs, KMT1A (also known as SUV39H1) and chromatin assembly factor 1 (CAF-1), respectively, contribute to both the immunogenicity of cancer cells and the lineage commitment and/or maturation of immune cells ${ }^{117,118}$; thus, epi-drugs can potentially be used to modulate antitumour immunity $^{119,120}$. Several mechanisms of resistance to immune-checkpoint inhibitors (ICIs) ${ }^{121}$ can be reverted, in preclinical models, by epigenetic manipulations (FIG. 3d) - a topic that we have reviewed elsewhere ${ }^{120}$. Here, we review important new data that have been reported in the past few months.

Preclinical modulation of adaptive immunity has been described with the ablation of LSD1 or inhibition of HDACs or DNMTs, which can enhance antigen presentation, in part through the induction of dsRNA production from endogenous retrovirus (ERV) genes and the resultant type I interferon response, leading to increased $\mathrm{T}$ cell infiltration of tumours and synergistic effects with ICIs $^{122-124}$. Similarly, DNMT inhibitors can further synergize with HDAC inhibitors to induce ERV transcription, promote CC-chemokine ligand 5 (CCL5)-dependent $\mathrm{T}$ cell infiltration and favour $\mathrm{T}$ cell memory and effector phenotypes, and to downregulate immunosuppressive MYC signalling, thereby reverting immune evasion in NSCLC models ${ }^{125}$. Interestingly, such an effect has also been described with CDK4/6 inhibitors, which indirectly reduce the activity of DNMT1 and thereby increase ERV expression $^{126}$; this observation highlights how some non-epigenetic drugs can have clinically relevant indirect epigenetic effects. Moreover, chemokine-dependent $\mathrm{T}$ cell attraction has also been described following enhanced expression of CXCL12 resulting from DNMT inhibition $^{127}$ or of CCL5, CXCL9 and CXCL10 from $\mathrm{LSD}^{128}$ or EZH2 inhibition ${ }^{129}$. Finally, the development of selective clinical-grade inhibitors of the histone-lysine $\mathrm{N}$-methyltransferase KMT1A might bring future clinical benefits via enhancement of the long-term memory reprogramming capacity of CD8 ${ }^{+} \mathrm{T}$ cells ${ }^{118}$.

With regard to innate immunity, LSD1, DNMT and HDAC inhibitors decrease the activity of MDSCs and promote an M1-like macrophage-mediated antitumour immune response in mouse models of breast or pancreatic cancer ${ }^{130,131}$. HDAC inhibition can also enhance the cytolytic activity of NK cells and TLR9 agonist-induced abscopal responses (that is, in lesions beyond the one treated with radiotherapy) $)^{132,133}$.

Of note, modulation of immunity using epi-drugs will probably require some fine-tuning, as illustrated by divergent results obtained with EZH2 inhibitors. Inhibition of EZH2 in T cells leads to enhanced antitumour responses with ICIs in preclinical models by eliciting functional alterations in regulatory $\mathrm{T}\left(\mathrm{T}_{\mathrm{reg}}\right)$ cells and by increasing the cytotoxicity of effector $\mathrm{T}$ cells ${ }^{134}$. In line with these results, a patient with $S M A R C B 1$-negative chordoma and disease progression on EZH2 inhibition with tazemetostat had a durable and exceptional abscopal response to radiotherapy lasting >2 years; comparisons of tumour biopsy samples obtained prior to treatment and during treatment with tazemetostat revealed a substantial increase in intratumoural and stromal infiltrates of $\mathrm{CD} 8^{+}$cytotoxic $\mathrm{T}$ cells and FOXP3 ${ }^{+} \mathrm{T}_{\text {reg }}$ cells, as well as enhanced expression of the immune-checkpoint proteins programmed cell death 1 (PD-1) and lymphocyte activation gene 3 (LAG3) on T cells ${ }^{135}$. These findings suggest that EZH2 inhibition can promote sustained antitumour immune responses and lead to immune-checkpoint activation $^{135}$, although the consequences of these effects, particularly with regard to ICI sensitivity, remain unclear at present. Contradictory pro-tumour effects have been reported preclinically with the EZH2 inhibitor GSK126, which resulted in increased numbers of MDSCs but decreased numbers of $\mathrm{CD} 4^{+} \mathrm{T}$ cells and IFN $\gamma^{+} \mathrm{CD}^{+} \mathrm{T}$ cells in the tumour microenvironment ${ }^{136}$. Together, these observations suggest that the cellular composition of the tumour and drug administration schedule can influence the immunomodulatory effects of EZH2 inhibition.

Some emerging clinical findings underscore the promise of combinations of epi-drugs and ICIs, notably in tumours that are resistant and/or refractory to ICIs (Supplementary Table 1). For example, the combination of the anti-PD-1 antibody pembrolizumab and vorinostat in a phase I/Ib study resulted in one confirmed partial response (PR) and eight stable disease responses among 24 patients with ICI-resistant metastatic NSCLC ${ }^{137}$. Moreover, preliminary results from the ENCORE-601 phase Ib/II trial evaluating the combination of pembrolizumab and entinostat demonstrate a PR in one patient with microsatellite-stable CRC (MSS-CRC), PRs in nine patients and a complete response in one patient with ICI-resistant melanoma and PRs in seven patients with ICI-resistant NSCLC (among 16, 53 and 67 patients, respectively $)^{138-140}$; in the NSCLC expansion cohort, a high level of peripheral CD $14^{+} \mathrm{CD} 16^{-} \mathrm{HLA}-\mathrm{DR}{ }^{\text {hi }}$ classic monocytes at baseline (defined as $\geq 13.1 \%$ of live PBMCs per millilitre of blood) was correlated with a better ORR (21.1\% versus $6.5 \%)$ and median PFS (5.3 months versus 2.7 months) ${ }^{138}$. Contrary to these single-arm phase I-II trials of HDAC inhibitors, however, the only randomized study with results reported to date revealed an increased risk of toxicities and no therapeutic benefit from combining pembrolizumab with the second-generation DNMT inhibitor CC-486 (oral azacytidine) ${ }^{141}$. The choice of full-dose CC-486 and the absence of patient selection, either for likely sensitivity to pembrolizumab or CC-486, might at least partially explain these results. Results from other studies of epi-drug plus ICI combinations are difficult to interpret at present because most data are preliminary and 
trials have predominantly been performed in ICI-naive patients; therefore, any responses observed might have been solely related to the ICI. In this context, comparisons with historical series of patients, preferably of OS outcomes, are necessary.

Of note, most combinations of epi-drugs and ICIs have been well tolerated (in contrast to many combinations of ICIs and targeted therapy ${ }^{142}$ ), even though most trials had simple designs with both agents used concomitantly, continuously and at full doses ${ }^{143,144}$ (Supplementary Table 1). Prolonged use of certain epi-drugs might, however, have deleterious effects on antitumour immune surveillance - for example, BET inhibitors can induce dramatic T cell depletion ${ }^{145}$. Thus, intermittent and/or sequential schedules that include an epigenetic 'priming' phase might be more effective owing to effects that foster a favourable tumour microenvironment for a response to ICIs. This approach is currently being tested clinically, for example, in a phase I study of pembrolizumab with the DNMT inhibitor azacitidine and/or the HDAC inhibitor romidepsin in patients with MSS-CRC $^{146}$ (NCT02512172; Supplementary Table 2).

\section{Optimizing epi-drug combinations}

A large number of clinical trials designed to investigate combinations of first-generation, second-generation or third-generation epi-drugs with various anticancer

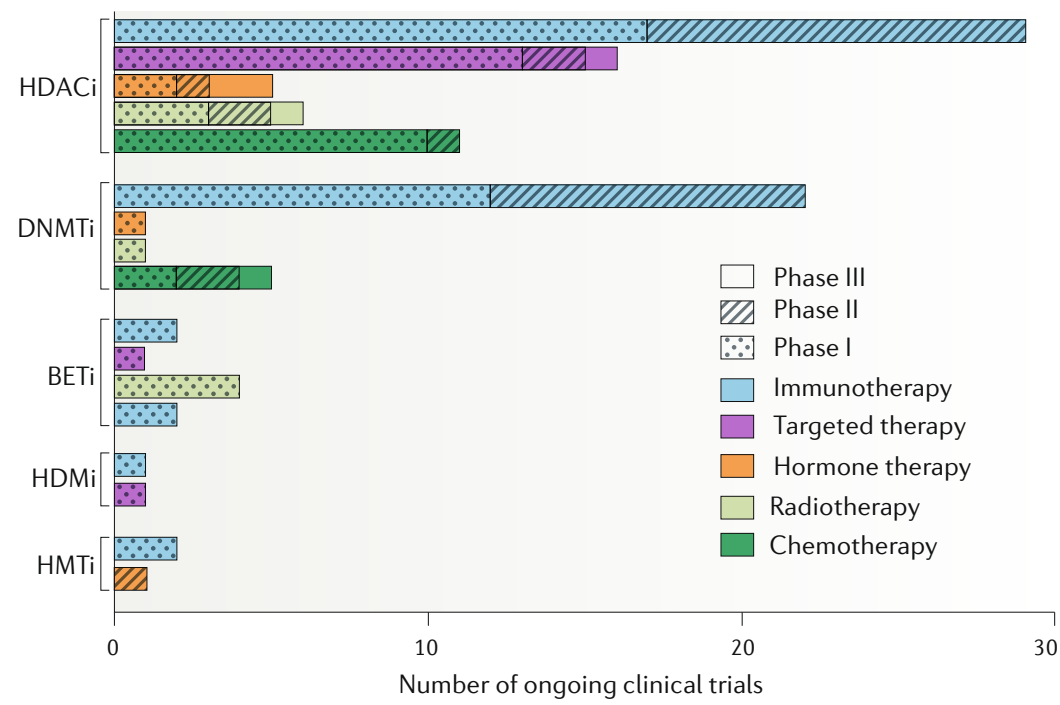

Fig. 4 | Overview of ongoing clinical trials of epi-drugs in combination with other anticancer therapies. The graph depicts the number of ongoing clinical trials of epigenetic drugs (epi-drugs) in combination with other anticancer therapies (based on searches of the ClinicalTrials.gov database; see Supplementary Table 2 for details), stratified by category of epi-drug and separated according to the key pillar of anticancer therapy to which the combination partner belongs; the relative numbers of phase I, phase II and phase III trials are also indicated by differential shading of each bar of the graph. DNA methyltransferase inhibitors (DNMTi) include azacytidine, decitabine and guadecitabine; histone deacetylase inhibitors (HDACi) include vorinostat, abexinostat, entinostat, ricolinostat, citarinostat, mocetinostat, epacadostat, panobinostat, romidepsin and nicotinamide; bromodomain and extra-terminal domain inhibitors (BETi) include INCB057643, PF-06821497, GS-5829, ZEN-3694, ABBV-075, molibresib and BMS-986158; histone methyltransferase inhibitors (HMTi) include enhancer of zeste homologue 2 inhibitors CPI-1205 and tazemetostat; and histone demethylases inhibitors (HDMi) include the lysine-specific histone demethylase $1 \mathrm{~A}$ inhibitors phenelzine and INCB059872. therapies are currently underway (FIG. 4). In the following sections of this Review, we outline the parameters that will be especially important to consider when interpreting the results of these trials as they become available and also for optimizing epi-drug development for combination therapy (FIG. 5a); we also discuss the challenges associated with each parameter and provide recommendations for designing future clinical trials.

\section{The medicinal chemistry of epi-drugs}

Optimal compound design is of crucial importance to the development of any drug, and is particularly relevant when multiple anticancer drugs are likely to be used in combination. As such, continued efforts to improve the medicinal chemistry of epi-drugs are essential. Some of the pharmacological characteristics that need to be improved include specificity (notably isoform selectivity), potency, bioavailability, stability at neutral $\mathrm{pH}$ and tumour accumulation (perhaps necessitating targeted tumour delivery), in order to limit the risk of adverse effects and increase the therapeutic index. Furthermore, many epigenetic regulators have multiple substrates, including histone and non-histone proteins, and thus have pleiotropic effects. For example, CBP and EP300 can acetylate not only histones $\mathrm{H} 3$ and H4, but also p53 (REF. $\left.{ }^{147}\right)$. Similarly, PRMT5 methylates arginine residues of histones $\mathrm{H} 2 \mathrm{~A}, \mathrm{H} 3$ and $\mathrm{H} 4$ as well as kinases, splicing factors and transcription factors, among other proteins ${ }^{148}$. In this context, designing a drug that selectively targets the addition of a particular post-translational modification to a single target protein becomes particularly difficult. Furthermore, epigenetic regulators often have multiple functional domains; the precise domain that should be targeted pharmacologically to obtain an anticancer effect can currently be tested using genome-editing technologies, such as CRISPR-Cas systems, to inform the selection of lead compounds $^{149}$.

With regard to the identification of lead compounds in epi-drug development, recapitulating and assessing the cellular effects of epigenetic regulators in vitro is not straightforward: many epigenetic enzymes act in the context of large multiprotein complexes, which can sometimes recruit additional and/or alternative cofactors or proteins; these interactions might not be faithfully reproduced in vitro, thereby modifying the preferred enzyme substrate and limiting the accuracy of assessments ${ }^{150}$. Similarly, in vitro assays based on nucleosome substrates cannot completely recapitulate the broad cellular effects of epi-drugs (especially when they are tissue-specific). High-quality antibodies that enable accurate detection of a specific post-translational modification on a given protein are also crucially lacking. Together, these challenges highlight the need for the development of additional research reagents, models and assays.

All these limitations should be considered when assessing the possible mechanisms of action of epi-drugs in clinical trials or preclinical studies. Notably, major progress in addressing these issues relating to the medicinal chemistry of epi-drugs has already been made during the development of first-generation, second-generation 
a

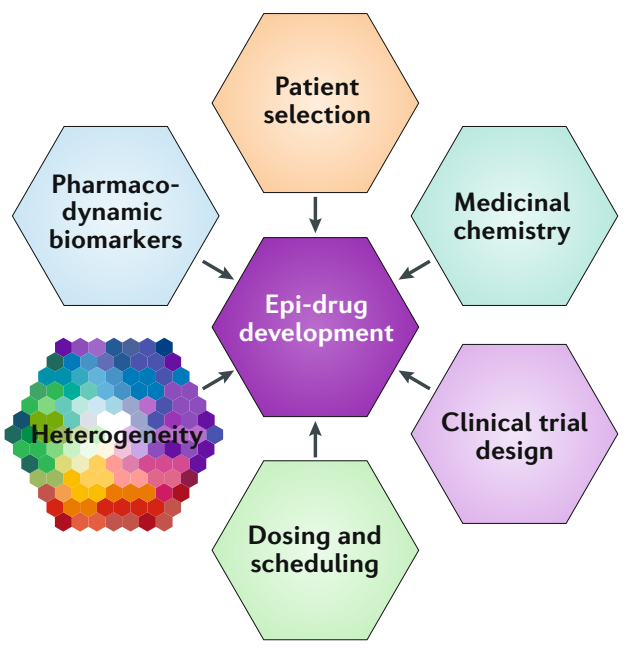

b

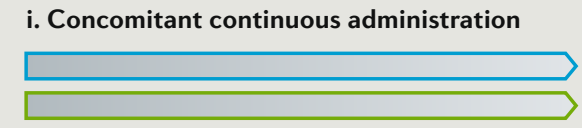

ii. Concomitant discontinuous administration

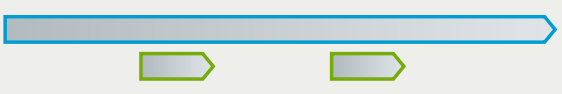

iii. Sequential discontinuous administration

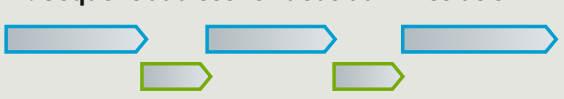

iv. Sequential continuous administration

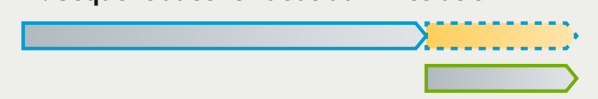

v. Epigenetic priming

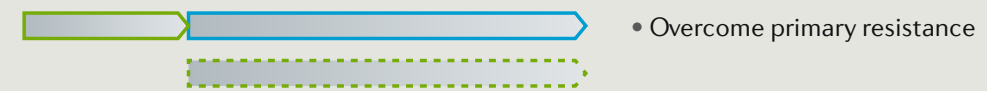

\begin{tabular}{lll}
\hline $\begin{array}{l}\text { Targeted, cytotoxic } \\
\text { and/or immunotherapy }\end{array}$ & $\begin{array}{l}\text { Next-generation targeted therapy } \\
\text { (or maybe continuation of original } \\
\text { targeted therapy) }\end{array}$ \\
\hline Epigenetic therapy & & $\begin{array}{l}\text { Continuous epigenetic } \\
\text { maintenance (may be optional) }\end{array}$
\end{tabular}

- Drug synergy or additivity

- Low toxicity combinations

- Drug synergy

- Medium or high toxicity combinations

- Overcome clonal epigenetic

resistance

- Modify sensitivity to

backbone therapy

- Alternate selection pressure

- Acquired epigenetic resistance

- Persistor cells/cancer stem cells

Overcome primary resistance 
have ever been investigated in clinical trials evaluating first-generation or second-generation compounds. By contrast, and learning from the aforementioned successes, third-generation epi-drugs have been shown to have efficacy as monotherapies in phase I studies involving molecularly enriched cohorts of patients selected according to the presence of certain sensitizing genomic alterations in the tumour (in particular, IDH1, EZH2 and BET inhibitors in patients with IDH1mutant AML, in patients with malignancies with $E Z H 2$, SMARCB1 or SMARCA4 aberrations, and in patients with BRD4-rearranged NUT midline carcinoma, respectively $)^{3-5}$. Nevertheless, only a few of the ongoing trials of epi-drugs are investigating predictive biomarkers for patient selection (Supplementary Table 2). In this context, comprehensive ancillary studies and molecular analyses of samples obtained from the enrolled patients are needed to facilitate biomarker discovery. Similarly, retrospective meta-analyses of available biomarker data should be planned. Unfortunately, the few exceptional responses and cases of resistance to epi-drugs that have been reported to date have mostly not been explored to determine the underlying mechanisms; such investigations might be very informative.

A number of factors might explain the limited implementation of biomarkers in trials of epi-drugs. First, the extent to which preclinical models recapitulate the clinical scenario is limited, not least because epigenetic marks vary according to cell culture conditions and are influenced by the conditions in the tumour microenvironment. Second, some epigenetic marks are cell type-specific and the particular cellular context in which epigenetic marks and genomic alterations occur is a crucial determinant of their functional consequences ${ }^{151}$. Therefore, similar to observations regarding the efficacy of BRAF inhibitors across different $B R A F$-mutant tumour types ${ }^{152}$, epi-drugs that target a given alteration might have different levels of efficacy across histotypes (for example, EZH2 inhibitors across different SMARCB1-deficient tumours or IDH inhibitors across various $I D H$-mutant haematological or solid malignancies); ongoing well-designed basket trials will provide further data on this issue (NCT02601950, NCT02746081 and NCT02481154). Third, analytically validated clinical grade assays are lacking for many potential epigenetic biomarkers. Clinical grade $B R C A 1$ or $M G M T$ promoter methylation assays exist, but were not designed for use in selecting patients for epi-drug therapy and have not been validated in this application. Furthermore, changes in histone or protein acetylation in response to HDAC inhibitors have been measured in various tissues (for example, PBMCs, B cells and tumour tissue) and the thresholds used to define the high versus low 'acetylator' phenotype have varied from study to study: the median value determined for the cohort of an individual study is usually applied retrospectively as the cut-off value in analyses of candidate predictive biomarkers ${ }^{56}$, with no clear rationale (one might argue that comparisons using the lower and upper quartiles would be more relevant). Indeed, to date, no proposed biomarkers of a response to epi-drugs have even been prospectively validated. Fourth, chromatin-based epigenetic analyses require large quantities of fresh or frozen material, although this hurdle might soon be overcome using new chromatin preparation methods (for example, Chrom-EX PE) ${ }^{153}$ that enable certain analyses, such as chromatin immunoprecipitation sequencing (ChIP-Seq), to be performed on smaller amounts of material from paraffin-embedded tissues. Finally, epigenetic marks and pharmacodynamic changes in epigenetic marks after treatment with epidrugs have predominantly been measured in PBMCs only, which might or might not reliably reflect changes occurring within the tumour ${ }^{27,56}$.

In this context, an important question exists regarding which selection biomarkers and corresponding techniques are most promising and should, therefore, be favoured for rational epi-drug development. Because DNA is a stable molecule and genomic alterations are well-characterized, it constitutes the primary material used in assessments of biomarkers for both epi-drug monotherapy and combination therapy. For example, genetic aberrations in SMARCA4 predict sensitivity to EZH2 inhibitors ${ }^{154}$, taxanes ${ }^{17}$ and PARP inhibitors ${ }^{155}$ as well as the EZH2 inhibitor plus TOP2 inhibitor combination in the setting of EGFR-mutant NSCLC ${ }^{19}$. Transcriptional signatures assessed using RNA-seq can also serve as a readout of chromatin state, including immune signatures in the context of epi-drug and ICI combinations, and can provide information on the expression of specific biomarkers of sensitivity and/or resistance (such as $B R D 4$ overexpression for sensitivity to BET inhibitors) $)^{156,157}$ or transcribed genomic alterations (for example, EZH2 mutations for EZH2 inhibitors $)^{4,158}$. Immunohistochemistry could potentially be used to more specifically assess functional biomarkers, such as H3K27me3 marks ${ }^{4}$, or to characterize features of the tumour microenvironment (such as immune infiltrates). Techniques that more specifically assess levels and patterns of DNA methylation (including bisulfite-sequencing) or chromatin accessibility (such as ATAC-Seq) $)^{159}$ should also be considered for the identification of future pharmacodynamic or predictive biomarkers ${ }^{160}$. Finally, because epigenetic changes are dynamic and reversible ${ }^{161}$, effort should be made to develop technologies that enable sequential longitudinal biomarker evaluation through the use of liquid biopsies of circulating materials, such as methylation of circulating tumour $\mathrm{DNA}^{162}$ or ATAC-Seq of DNA from circulating tumour cells ${ }^{160}$. Furthermore, with the advent of technologies for single-cell assessments, the ability to identify epigenetic markers in heterogeneous cell populations will be a major asset, for example, in predicting tumour cell heterogeneity that might preclude or limit a response and in defining specific cell types that are affected (for example, particular types of immune cells in the context of epi-drug and ICI combinations) ${ }^{163}$.

\section{Drug administration strategies}

Most epi-drug combinations have been hampered by the occurrence of acute or chronic DLTs. A single-centre retrospective study revealed that $66 \%$ of epi-drugrelated toxicities of grade 3 or 4 occurred after cycle 1 , suggesting that extending the assessment period for DLTs beyond cycle 1 is important ${ }^{28,105,164}$. Treatment with 
certain epi-drugs can achieve the desired pharmacodynamic modulation at doses below the maximum tolerated $\operatorname{lose}^{27}$; therefore, the use of lower doses should be considered if possible. For example, increasing the dosage of tazemetostat from $100 \mathrm{mg}$ to $800 \mathrm{mg}$ twice daily reduced $\mathrm{H} 3 \mathrm{~K} 27 \mathrm{me} 3$ levels in the stratum spinosum layer of the skin, with modelling studies suggesting a maximum inhibitory effect at $800 \mathrm{mg}$ twice daily; further increasing the dose to $1,600 \mathrm{mg}$ twice daily had almost no additional effect ${ }^{4}$. Similarly, histone acetylation was maximally inhibited at an abexinostat dose of $30 \mathrm{mg} / \mathrm{m}^{2}$ twice daily, with only limited further inhibition observed at higher doses ${ }^{28}$. Intermittent dosing might be an alternative approach to limiting toxicities ${ }^{104}$. Of note, dosage and scheduling might be especially important with epi-drug plus ICI combinations, as both therapeutic strategies can be beneficial or detrimental depending on the dose, schedule and cellular context ${ }^{141,165,166}$.

Treatment sequence and schedule can also be varied according to the main desired anticancer effect of the epi-drug - that is, whether the primary goal is to exploit synergy whilst avoiding prohibitive toxicities, apply alternating selective pressures, overcome intrinsic resistance or reverse acquired resistance (FIG. 5b). This flexibility in trial design is now being implemented in certain clinical studies, such as randomized phase I trials combining epidrug 'priming' to increase the sensitivity of tumours to ICIs (for example, NCT02512172; Supplementary Table 2). Importantly, reports indicate that tumours resistant and/or refractory to a certain therapy can be re-sensitized after addition of an epi-drug to the therapy (for example, re-sensitization to PD-1 inhibitors or to pazopanib when combined with HDAC inhibition in patients with NSCLC $^{138,167}$ and those with RCC ${ }^{168}$, respectively). Whether sequential treatment, whereby epi-drug therapy would precede re-exposure to the agent of interest, would also result in some re-sensitization is unknown and warrants investigation. Therefore, long-term follow-up investigations that include the recording of responses to subsequent lines of therapy could be considered in trials involving epi-drugs, whenever possible.

\section{Trial design and response criteria}

Similar to observations with IDH inhibitors and BET inhibitors, which can require several months to induce responses in patients with haematological malignan$\operatorname{cies}^{3,169}$, EZH2 inhibitors have sometimes led to delayed responses in patients with solid tumours ${ }^{4}$. Indeed, the time frame required for epi-drugs to rewire transcriptional programmes and thereby induce cell differentiation or phenotypic changes in clinical tissues is not yet clear. In this context, evaluation criteria such as tumour growth rate, in addition to clinical benefit in terms of prolonged objective responses or stable disease, should be considered in clinical trial protocols when preclinical data suggest gradual epigenetic effects of the treatment that is under investigation. This strategy could prevent patients who would be expected to benefit from the delayed anticancer effects from being prematurely taken off study owing to slow disease progression, according to the traditional Response Evaluation Criteria in Solid Tumors (RECIST).

\section{Epigenetic heterogeneity}

Epigenetic marks are labile - that is, dynamically altered much more easily than DNA sequences - and in most cases are tissue-specific; therefore, epigenetic heterogeneity is a major therapeutic challenge that drives both dissociated responses ${ }^{170}$ (that is, regression of some lesions but not others during therapy) and acquired resistance ${ }^{171}$. In this context, multi-region sampling of a single lesion and/or biopsy sampling of multiple lesions might be informative or even necessary. The exponential current development of single-cell sequencing technologies, including scRNA-seq, scChIP-Seq and scATAC-Seq, will help to resolve this particular issue ${ }^{163,172}$; although such approaches still present technical, bioinformatic and computational difficulties, they might ultimately transform our understanding of tumour evolution, heterogeneity and epigenetic plasticity, and how these phenomena affect treatment response.

\section{Paediatric populations}

Paediatric cancers are often driven by epigenetic dysregulation that is the result of, for example, mutations in chromatin remodelling genes (such as SMARCB1 in malignant rhabdoid tumours), 'oncohistones' (such as oncogenic histone $\mathrm{H} 3$ alterations in diffuse intrinsic pontine gliomas) or aberrations affecting transcription factors (including EWS-FLI1 fusions in Ewing sarcoma $)^{2,173,174}$. Therefore, the development of epi-drug combinations holds particular promise for the treatment of children and young adults, populations in which other anticancer treatments, such as radiotherapy and cytotoxic chemotherapies, can have especially problematic long-term toxicities.

\section{Conclusions}

A robust body of mechanistic evidence supports the hypothesis that epi-drugs can synergize with other anticancer agents and reverse therapy resistance in preclinical models. Nevertheless, the efficacy of epi-drugs tested in clinical trials to date has been a clear disappointment. The limited tolerability of most combinations comprising epi-drugs and cytotoxic therapies is a major challenge, but the exploration of lower doses, sequential scheduling and targeted delivery of epi-drugs might considerably improve the therapeutic index. We regret, however, that most studies are still being designed according to an outdated 'one size fits all' approach and crucially lack predictive biomarkers for patient selection. This situation is clearly suboptimal: epi-drugs are becoming increasingly specific for their target enzymes and, thus, their development should follow a precision-medicine approach. As a testament to this concept, an increasing number of early phase studies assessing novel epi-drugs as single agents are now following this rational, biomarker-directed approach (for example, NCT02601950, NCT02746081 and NCT02481154) and might pave the path to success. We hope that, together with the broadening characterization of the genetic and epigenetic landscapes of tumours, this strategy will finally enable us to unleash the full potential of epi-drugs.

Published online: 30 September 2019 
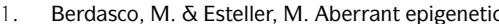 landscape in cancer: how cellular identity goes awry. Dev. Cell 19, 698-711 (2010).

2. Morel, D., Almouzni, G., Soria, J.-C. \& Postel-Vinay, S. Targeting chromatin defects in selected solid tumors based on oncogene addiction, synthetic lethality and epigenetic antagonism. Ann. Oncol. 28, 254-269 (2017)

3. DiNardo, C. D. et al. Durable remissions with ivosidenib in IDH1-mutated relapsed or refractory AML. N. Engl. J. Med. 378, 2386-2398 (2018).

4. Italiano, A. et al. Tazemetostat, an EZH2 inhibitor, in relapsed or refractory B-cell non-Hodgkin lymphoma and advanced solid tumours: a first-in-human open-label, phase 1 study. Lancet Oncol. 19. 649-659 (2018)

5. Stathis, A. et al. Clinical response of carcinomas harboring the BRD4-NUT oncoprotein to the targeted bromodomain inhibitor OTX015/MK-8628. Cancer Discov. 6, 492-500 (2016).

6. Easwaran, H., Tsai, H.-C. \& Baylin, S. B. Cancer epigenetics: tumor heterogeneity, plasticity of stem-like states, and drug resistance. Mol. Cell 54 716-727 (2014)

7. Shaffer, S. M. et al. Rare cell variability and drug-induced reprogramming as a mode of cancer drug resistance. Nature 546, 431-435 (2017)

8. Gupta, P. B., Pastushenko, I., Skibinski, A., Blanpain, C $\&$ Kuperwasser, C. Phenotypic plasticity: driver of cancer initiation, progression, and therapy resistance. Cell Stem Cell 24, 65-78 (2019).

9. Hosseini, A. \& Minucci, S. A comprehensive review of lysine-specific demethylase 1 and its roles in cancer. Epigenomics 9, 1123-1142 (2017).

10. Hanahan, D. \& Weinberg, R. A. Hallmarks of cancer the next generation. Cell 144, 646-674 (2011).

11. Mohammad, H. P., Barbash, O. \& Creasy, C. L. Targeting epigenetic modifications in cancer therapy: erasing the roadmap to cancer. Nat. Med. $\mathbf{2 5}$ 403-418 (2019)

12. Bruzzese, F. et al. Synergistic antitumor effect between vorinostat and topotecan in small cell lung cancer cells is mediated by generation of reactive oxygen species and DNA damage-induced apoptosis. Mol. Cancer Ther. 8, 3075-3087 (2009)

13. Thurn, K. T., Thomas, S., Raha, P., Qureshi, I. \& Munster, P. N. Histone deacetylase regulation of ATM-mediated DNA damage signaling. Mol. Cancer Ther. 12, 2078-2087 (2013).

14. Zhang, Y.-W. et al. Integrated analysis of DNA methylation and mRNA expression profiling reveals candidate genes associated with cisplatin resistance in non-small cell lung cancer. Epigenetics 9, 896-909 (2014).

15. Candelaria, M. et al. A phase II study of epigenetic therapy with hydralazine and magnesium valproate to overcome chemotherapy resistance in refractory solid tumors. Ann. Oncol. 18, 1529-1538 (2007)

16. Wang, N. et al. TGFBI promoter hypermethylation correlating with paclitaxel chemoresistance in ovarian cancer. J. Exp. Clin. Cancer Res. 31, 6 (2012).

17. Gurard-Levin, Z. A. et al. Chromatin regulators as a guide for cancer treatment choice. Mol. Cancer Ther 15, 1768-1777 (2016).

18. Bell, E. H. et al. SMARCA4/BRG1 is a novel prognostic biomarker predictive of cisplatin-based chemotherapy outcomes in resected non-small cell lung cancer. Clin. Cancer Res. 22, 2396-2404 (2016).

19. Fillmore, C. M. et al. EZH2 inhibition sensitizes BRG1 and EGFR mutant lung tumours to Topoll inhibitors. Nature 520, 239-242 (2015)

20. Doroshow, D. B., Eder, J. P. \& LoRusso, P. M. BET inhibitors: a novel epigenetic approach. Ann. Oncol. 28, 1776-1787 (2017).

21. Bajbouj, K. et al. PRMT5 selective inhibitor enhances therapeutic efficacy of cisplatin in lung adenocarcinoma cells [abstract]. Cancer Res. 79 (Suppl 13), 4711 (2019).

22. Balch, C. et al. Antimitogenic and chemosensitizing effects of the methylation inhibitor zebularine in ovarian cancer. Mol. Cancer Ther. 4, 1505-1514 (2005).

23. Zeller, C. et al. Candidate DNA methylation drivers of acquired cisplatin resistance in ovarian cancer identified by methylome and expression profiling. Oncogene 31, 4567-4576 (2012).

24. Glasspool, R. M. et al. A randomised, phase II trial of the DNA-hypomethylating agent 5-aza-2'deoxycytidine (decitabine) in combination with carboplatin vs carboplatin alone in patients with recurrent, partially platinum-sensitive ovarian cancer. Br. J. Cancer 110, 1923-1929 (2014).
25. Matei, D. et al. Epigenetic resensitization to platinum in ovarian cancer. Cancer Res. 72, 2197-2205 (2012).

26. Kwon, N.-H., Kim, J.-S., Lee, J.-Y., Oh, M.-J. \& Choi, D.-C. DNA methylation and the expression of IL-4 and IFN- $\gamma$ promoter genes in patients with bronchial asthma. J. Clin. Immunol. 28, 139-146 (2008).

27. Appleton, K. et al. Phase I and pharmacodynamic trial of the DNA methyltransferase inhibitor decitabine and carboplatin in solid tumors. J. Clin. Oncol. 25 , 4603-4609 (2007).

28. Choy, E. et al. Phase 1 study of oral abexinostat, a histone deacetylase inhibitor, in combination with doxorubicin in patients with metastatic sarcoma. Cancer 121, 1223-1230 (2015).

29. Nogales, V. et al. Epigenetic inactivation of the putative DNA/RNA helicase SLFN11 in human cancer confers resistance to platinum drugs. Oncotarget 7 , 3084-3097 (2016).

30. Giovannetti, E. et al. Role of proton-coupled folate transporter in pemetrexed resistance of mesothelioma: clinical evidence and new pharmacological tools. Ann. Oncol. 28, 2725-2732 (2017).

31. Camphausen, K. \& Tofilon, P. J. Inhibition of histone deacetylation: a strategy for tumor radiosensitization. J. Clin. Oncol. 25, 4051-4056 (2007).

32. Zhang, Y. et al. Attenuated DNA damage repair by trichostatin A through BRCA1 suppression. Radiat. Res. 168, 115-124 (2007).

33. Zhu, X., Wang, Y., Tan, L. \& Fu, X. The pivotal role of DNA methylation in the radio-sensitivity of tumor radiotherapy. Cancer Med 7, 3812-3819 (2018).

34. Wu, C. et al. Inhibition of EZH2 by chemo- and radiotherapy agents and small molecule inhibitors induces cell death in castration-resistant prostate cancer. Oncotarget 7, 3440-3452 (2016).

35. Xia, $\mathrm{H}$. et al. EZH2 silencing with RNAi enhances irradiation-induced inhibition of human lung cancer growth in vitro and in vivo. Oncol. Lett. 4, 135-140 (2012).

36. Chinnaiyan, P. et al. Postradiation sensitization of the histone deacetylase inhibitor valproic acid. Clin. Cancer Res. 14, 5410-5415 (2008).

37. Wang, J. et al. Pharmacological targeting of BET proteins attenuates radiation-induced lung fibrosis. Sci. Rep. 8, 998 (2018)

38. Chan, E. et al. Phase I trial of vorinostat added to chemoradiation with capecitabine in pancreatic cancer. Radiother. Oncol. 119, 312-318 (2016).

39. DuBois, S. G. et al. Phase I study of vorinostat as a radiation sensitizer with 1311-metaiodobenzylguanidine (131I-MIBG) for patients with relapsed or refractory neuroblastoma. Clin. Cancer Res. 21, 2715-2721 (2015).

40. Galanis, E. et al. Phase $\mathrm{I} / \mathrm{II}$ trial of vorinostat combined with temozolomide and radiation therapy for newly diagnosed glioblastoma: results of Alliance N0874 ABTC 02. Neuro. Oncol. 20, 546-556 (2018).

41. Whitesell, L. \& Lindquist, S. L. HSP90 and the chaperoning of cancer. Nat. Rev. Cancer 5, 761-772 (2005).

42. Welboren, W-J. et al. ChIP-Seq of ERalpha and RNA polymerase II defines genes differentially responding to ligands. EMBO J. 28, 1418-1428 (2009).

43. Hodges-Gallagher, L., Valentine, C. D., Bader, S. E. \& Kushner, P. J. Inhibition of histone deacetylase enhances the anti-proliferative action of antiestrogens on breast cancer cells and blocks tamoxifen-induced proliferation of uterine cells. Breast Cancer Res. Treat. 105, 297-309 (2007).

44. Thomas, S., Thurn, K. T., Biçaku, E., Marchion, D. C \& Münster, P. N. Addition of a histone deacetylase inhibitor redirects tamoxifen-treated breast cancer cells into apoptosis, which is opposed by the induction of autophagy. Breast Cancer Res. Treat. 130 , 437-447 (2011)

45. Alao, J. P. et al. Histone deacetylase inhibitor trichostatin A represses estrogen receptor alphadependent transcription and promotes proteasoma degradation of cyclin D1 in human breast carcinoma cell lines. Clin. Cancer Res. 10, 8094-8104 (2004)

46. Margueron, R. et al. Histone deacetylase inhibition and estrogen receptor alpha levels modulate the transcriptional activity of partial antiestrogens. J. Mol. Endocrinol. 32, 583-594 (2004).

47. Reid, G. et al. Multiple mechanisms induce transcriptional silencing of a subset of genes, including oestrogen receptor alpha, in response to deacetylase inhibition by valproic acid and trichostatin A. Oncogene 24, 4894-4907 (2005)

48. Fiskus, W. et al. Hydroxamic acid analogue histone deacetylase inhibitors attenuate estrogen receptor-alpha levels and transcriptional activity: a result of hyperacetylation and inhibition of chaperone function of heat shock protein 90. Clin. Cancer Res. 13, 4882-4890 (2007).

49. Dowling, C. et al. Discovery of a novel histone deacetylase 6 inhibitor that kills drug-resistant breast cancer [abstract]. Cancer Res. 79 (Suppl 13), 5186 (2019).

50. Feng, Q. et al. An epigenomic approach to therapy for tamoxifen-resistant breast cancer. Cell Res. $\mathbf{2 4}$ 809-819 (2014).

51. Ladd, B. et al. Effective combination therapies in preclinical endocrine resistant breast cancer models harboring ER mutations. Oncotarget 7, 54120-54136 (2016).

52. Sengupta, S., Biarnes, M. C., Clarke, R. \& Jordan, V. C Inhibition of BET proteins impairs estrogen-mediated growth and transcription in breast cancers by pausing RNA polymerase advancement. Breast Cancer Res. Treat. 150, 265-278 (2015).

53. Nagarajan, S. et al. Bromodomain protein BRD4 is required for estrogen receptor-dependent enhancer activation and gene transcription. Cell Rep. 8 , 460-469 (2014).

54. Bommi-Reddy, A. et al. Efficacy of a novel EP300/CBP histone acetyltransferase inhibitor in hormone responsive breast cancer [abstract]. Cancer Res 79 (Suppl 13), 4722 (2019).

55. Munster, P. N. et al. A phase II study of the histone deacetylase inhibitor vorinostat combined with tamoxifen for the treatment of patients with hormone therapy-resistant breast cancer. Br. J. Cancer 104 , 1828-1835 (2011).

56. Yardley, D. A. et al. Randomized phase II, double-blind, placebo-controlled study of exemestane with or without entinostat in postmenopausal women with locally recurrent or metastatic estrogen receptor-positive breast cancer progressing on treatment with a nonsteroidal aromatase inhibitor. J. Clin. Oncol. 31, 2128-2135 (2013).

57. Tomita, Y. et al. The interplay of epigenetic therapy and immunity in locally recurrent or metastatic estrogen receptor-positive breast cancer: correlative analysis of ENCORE 301, a randomized, placebo-controlled phase II trial of exemestane with or without entinostat. Oncoimmunology 5, e1219008 (2016).

58. Yeruva, S. L. H. et al. E2112: randomized phase III trial of endocrine therapy plus entinostat/placebo in patients with hormone receptor-positive advanced breast cancer. npj Breast Cancer 4, 1-5 (2018).

59. Jiang, Z. et al. Tucidinostat plus exemestane for postmenopausal patients with advanced, hormone receptor-positive breast cancer (ACE): a randomised, double-blind, placebo-controlled, phase 3 trial. Lancet Oncol. 20, 806-815 (2019).

60. Gaughan, L., Logan, I. R., Cook, S., Neal, D. E. \& Robson, C. N. Tip60 and histone deacetylase 1 regulate androgen receptor activity through changes to the acetylation status of the receptor. J. Biol. Chem 277, 25904-25913 (2002).

61. Xia, Q. et al. Chronic administration of valproic acid inhibits prostate cancer cell growth in vitro and in vivo. Cancer Res. 66, 7237-7244 (2006).

62. Urbanucci, A., Marttila, S., Jänne, O. A. \& Visakorpi, T. Androgen receptor overexpression alters binding dynamics of the receptor to chromatin and chromatin structure. Prostate 72, 1223-1232 (2012).

63. Jansson, K. H. et al. High-throughput screens identify HSP90 inhibitors as potent therapeutics that target inter-related growth and survival pathways in advanced prostate cancer. Sci. Rep. 8, 17239 (2018).

64. Asangani, I. A. et al. BET bromodomain inhibitors enhance efficacy and disrupt resistance to AR antagonists in the treatment of prostate cancer. Mol. Cancer Res. 14, 324-331 (2016).

65. Asangani, I. A. et al. Therapeutic targeting of BET bromodomain proteins in castration-resistant prostate cancer. Nature 510, 278-282 (2014).

66. Ferrari, A. C. et al. Epigenetic therapy with panobinostat combined with bicalutamide rechallenge in castration-resistant prostate cancer. Clin. Cancer Res. 25, 52-63 (2019).

67. Edwards, A., Li, J., Atadja, P., Bhalla, K. \& Haura, E. B. Effect of the histone deacetylase inhibitor LBH589 against epidermal growth factor receptor-dependent human lung cancer cells. Mol. Cancer Ther. 6 , 2515-2524 (2007).

68. Ng, K. P. et al. A common BIM deletion polymorphism mediates intrinsic resistance and inferior responses to tyrosine kinase inhibitors in cancer. Nat. Med. 18 , 521-528 (2012). 
69. Tanimoto, A. et al. Histone deacetylase 3 inhibition overcomes BIM deletion polymorphism-mediated osimertinib resistance in EGFR-mutant lung cancer. Clin. Cancer Res. 23, 3139-3149 (2017).

70. Stuhlmiller, T. J. et al. Inhibition of lapatinib-induced kinome reprogramming in ERBB2-positive breast cancer by targeting BET family bromodomains. Cell Rep. 11, 390-404 (2015)

71. Stuhlmiller, T. J., Miller, S. M. \& Johnson, G. L. Epigenetic inhibition of adaptive bypass responses to lapatinib by targeting BET bromodomains. Mol. Cell. Oncol. 3 (2016).

72. Leonard, B. et al. BET inhibition overcomes receptor tyrosine kinase-mediated cetuximab resistance in HNSCC. Cancer Res. 78, 4331-4343 (2018).

73. Witta, S. E. et al. Randomized phase II trial of erlotinib with and without entinostat in patients with advanced non-small-cell lung cancer who progressed on prior chemotherapy. J. Clin. Oncol. 30, 2248-2255 (2012)

74. Han, J.-Y. et al. Phase I/II study of gefitinib (Iressa( $\left.{ }^{(}\right)$) and vorinostat (IVORI) in previously treated patients with advanced non-small cell lung cancer. Cancer Chemother. Pharmacol. 75, 475-483 (2015).

75. Recondo, G., Facchinetti, F., Olaussen, K. A., Besse, B. $\&$ Friboulet, L. Making the first move in EGFR-driven or ALK-driven NSCLC: first-generation or next-generation TKI? Nat Rev Clin Oncol 15, 694-708 (2018).

76. Oian, D. Z. et al. Class II histone deacetylases are associated with VHL-independent regulation of hypoxia-inducible factor 1 alpha. Cancer Res. 66 8814-8821 (2006)

77. Qian, D. Z. et al. The histone deacetylase inhibito NVP-LAQ824 inhibits angiogenesis and has a greater antitumor effect in combination with the vascular endothelial growth factor receptor tyrosine kinase inhibitor PTK787/ZK222584. Cancer Res. 64, 6626-6634 (2004).

78. Ellis, L., Hammers, H. \& Pili, R. Targeting tumor angiogenesis with histone deacetylase inhibitors. Cancer Lett. 280, 145-153 (2009).

79. Pili, R. et al. Combination of the histone deacetylase inhibitor vorinostat with bevacizumab in patients with clear-cell renal cell carcinoma: a multicentre, single-arm phase I/II clinical trial. Br. J. Cancer 116 , 874-883 (2017)

80. Bitzer, M. et al. Resminostat plus sorafenib as second-line therapy of advanced hepatocellular carcinoma - the SHELTER study. J. Hepatol. 65 280-288 (2016).

81. Drappatz, J. et al. Phase I study of panobinostat in combination with bevacizumab for recurrent high-grade glioma. J. Neurooncol. 107, 133-138 (2012).

82. Lee, E. Q. et al. Phase II study of panobinostat in combination with bevacizumab for recurrent glioblastoma and anaplastic glioma. Neuro. Oncol. 17, 862-867 (2015)

83. Aggarwal, R. et al. Inhibiting histone deacetylase as a means to reverse resistance to angiogenesis inhibitors: phase I study of abexinostat plus pazopanib in advanced solid tumor malignancies. J. Clin. Oncol. 35, 1231-1239 (2017).

84. Chen, C. S., Weng, S. C., Tseng, P. H., Lin, H. P. \& Chen, C. S. Histone acetylation-independent effect of histone deacetylase inhibitors on Akt through the reshuffling of protein phosphatase 1 complexes. J. Biol. Chem. 280, 38879-38887 (2005)

85. Bali, P. et al. Inhibition of histone deacetylase 6 acetylates and disrupts the chaperone function of heat shock protein 90: a novel basis for antileukemia activity of histone deacetylase inhibitors. J. Biol. Chem. 280, 26729-26734 (2005).

86. Mahalingam, D. et al. Vorinostat enhances the activity of temsirolimus in renal cell carcinoma through suppression of survivin levels. Clin. Cancer Res. 16, 141-153 (2010)

87. Malone, C. F. et al. mTOR and HDAC inhibitors converge on the TXNIP/thioredoxin pathway to cause catastrophic oxidative stress and regression of RAS-driven tumors. Cancer Discov. 7, 1450-1463 (2017).

88. Piao, J. et al. Superior efficacy of co-treatment with the dual PI3K/mTOR inhibitor BEZ235 and histone deacetylase inhibitor trichostatin A against NSCLC. Oncotarget 7, 60169-60180 (2016)

89. Lin, T.-W. et al. TDP-43/HDAC6 axis promoted tumor progression and regulated nutrient deprivation-induced autophagy in glioblastoma. Oncotarget 8 , 56612-56625 (2017)

90. Pei, Y. et al. HDAC and PI3K Antagonists cooperate to inhibit growth of MYC-driven medulloblastoma. Cancer Cell 29, 311-323 (2016).
91. Vázquez, R. et al. The bromodomain inhibitor OTX015 (MK-8628) exerts anti-tumor activity in triplenegative breast cancer models as single agent and in combination with everolimus. Oncotarget $\mathbf{8}$, 7598-7613 (2016).

92. Stratikopoulos, E. E. et al. Kinase and BET inhibitors together clamp inhibition of PI3K signaling and overcome resistance to therapy. Cancer Cell 27 837-851 (2015)

93. Iniguez, A. B. et al. Resistance to epigenetic-targeted therapy engenders tumor cell vulnerabilities associated with enhancer remodeling. Cancer Cell 34, 922-938 (2018).

94. Kurimchak, A. M. et al. Resistance to BET bromodomain inhibitors is mediated by kinome reprogramming in ovarian cancer. Cell Rep. 16 1273-1286 (2016)

95. Gyawali, B. \& Prasad, V. Drugs that lack single-agent activity: are they worth pursuing in combination? Nat. Rev. Clin. Oncol. 14, 193-194 (2017).

96. Zibelman, M. et al. Phase I study of the mTOR inhibitor ridaforolimus and the HDAC inhibitor vorinostat in advanced renal cell carcinoma and other solid tumors. Invest. New Drugs 33, 1040-1047 (2015).

97. Park, H. et al. Phase I dose-escalation study of the mTOR inhibitor sirolimus and the HDAC inhibitor vorinostat in patients with advanced malignancy. Oncotarget 7, 67521-67531 (2016).

98. Carson, R. et al. HDAC Inhibition overcomes acute resistance to MEK inhibition in -mutant colorectal cancer by downregulation of c-FLIPL. Clin. Cancer Res. 21, 3230-3240 (2015)

99. Gallagher, S. J. et al. HDAC inhibitors restore BRAF-inhibitor sensitivity by altering PI3K and survival signalling in a subset of melanoma. Int. J. Cancer 142 1926-1937 (2018)

100. Johannessen, C. M. et al. A melanocyte lineage program confers resistance to MAP kinase pathway inhibition. Nature 504, 138-142 (2013).

101. Maertens, O. et al. MAPK pathway suppression unmasks latent DNA repair defects and confers a chemical synthetic vulnerability in BRAF-, NRAS-, and NF1-mutant melanomas. Cancer Discov, 9, 526-545 (2019).

102. Paoluzzi, L. et al. BET and BRAF inhibitors act synergistically against BRAF-mutant melanoma. Cancer Med. 5, 1183-1193 (2016).

103. Fallahi-Sichani, M. et al. Adaptive resistance of melanoma cells to RAF inhibition via reversible induction of a slowly dividing de-differentiated state. Mol. Syst. Biol. 13, 905 (2017)

104. Kitajima, S. et al. Overcoming resistance to dual innate immune and MEK inhibition downstream of KRAS. Cancer Cell 34, 439-452 (2018).

105. Postel-Vinay, S. et al. Phase I trials of molecularly targeted agents: should we pay more attention to late toxicities? J. Clin. Oncol. 29, 1728-1735 (2011).

106. Paoletti, X. et al. Defining dose-limiting toxicity for phase 1 trials of molecularly targeted agents: results of a DLT-TARGETT international survey. Eur. J. Cancer 50, 2050-2056 (2014)

107. Ha, K. et al. Histone deacetylase inhibitor treatment induces 'BRCAness' and synergistic lethality with PARP inhibitor and cisplatin against human triple negative breast cancer cells. Oncotarget 5, 5637-5650 (2014).

108. Yin, L. et al. PARP inhibitor veliparib and HDAC inhibitor SAHA synergistically co-target the UHRF $1 /$ BRCA1 DNA damage repair complex in prostate cancer cells. J. Exp. Clin. Cancer Res. 37, 153 (2018)

109. Konstantinopoulos, P. A., Wilson, A. J., Saskowski, J., Wass, E. \& Khabele, D. Suberoylanilide hydroxamic acid (SAHA) enhances olaparib activity by targeting homologous recombination DNA repair in ovarian cancer. Gynecol. Oncol. 133, 599-606 (2014).

110. Karakashev, S. et al. BET bromodomain inhibition synergizes with PARP inhibitor in epithelial ovarian cancer Cell Rep. 21, 3398-3405 (2017).

111. Sun, C. et al. BRD4 inhibition is synthetic lethal with PARP inhibitors through the induction of homologous recombination deficiency. Cancer Cell 33, 401-416 (2018).

112. Wilson, A. J., Stubbs, M., Liu, P., Ruggeri, B. \& Khabele, D. The BET inhibitor INCB054329 reduces homologous recombination efficiency and augments PARP inhibitor activity in ovarian cancer. Gynecol. Oncol. 149, 575-584 (2018).

113. Yang, L. et al. Repression of BET activity sensitizes homologous recombination-proficient cancers to PARP inhibition. Sci. Transl Med. 9, eaal 1645 (2017).

114. Pawar, A., Gollavilli, P. N., Wang, S. \& Asangani, I. A Resistance to BET inhibitor leads to alternative therapeutic vulnerabilities in castration-resistant prostate cancer. Cell Rep. 22, 2236-2245 (2018).

115. Huang, X. et al. Targeting epigenetic crosstalk as a therapeutic strategy for EZH2-aberrant solid tumors. Cell 175, 186-199 (2018).

116. Agrawal, K. et al. Chromatin reader machinery as target for overcoming resistance to DNA-demethylating epi-drug decitabine [abstract]. Cancer Res. 79 (Suppl 13), 5182 (2019).

117. Ng, C. et al. The histone chaperone CAF-1 cooperates with the DNA methyltransferases to maintain $\mathrm{Cd} 4$ silencing in cytotoxic T cells. Genes Dev. 33, 669-683 (2019).

118. Pace, L. et al. The epigenetic control of stemness in CD8+ T cell fate commitment. Science 359, 177-186 (2018).

119. Lau, C. M. et al. Epigenetic control of innate and adaptive immune memory. Nat. Immunol. 19, 963-972 (2018)

120. Aspeslagh, S., Morel, D., Soria, J.-C. \& Postel-Vinay, S. Epigenetic modifiers as new immunomodulatory therapies in solid tumours. Ann. Oncol. 29, 812-824 (2018).

121. Sharma, P., Hu-Lieskovan, S., Wargo, J. A. \& Ribas, A Primary, adaptive, and acquired resistance to cancer immunotherapy. Cell 168, 707-723 (2017).

122. Stone, M. L. et al. Epigenetic therapy activates type I interferon signaling in murine ovarian cancer to reduce immunosuppression and tumor burden. Proc. Natl Acad. Sci. USA 114, E10981-E10990 (2017).

123. Sheng, W. et al. LSD1 ablation stimulates anti-tumor immunity and enables checkpoint blockade. Cell 174 549-563 (2018)

124. Terranova-Barberio, M. et al. HDAC inhibition potentiates immunotherapy in triple negative breast cancer. Oncotarget 8, 114156-114172 (2017).

125. Topper, M. J. et al. Epigenetic therapy ties MYC depletion to reversing immune evasion and treating lung cancer. Cell 171, 1284-1300 (2017).

126. Goel, S. et al. CDK4/6 inhibition triggers anti-tumour immunity. Nature 548, 471-475 (2017).

127. Li, B. et al. Epigenetic regulation of CXCL12 plays a critical role in mediating tumor progression and the immune response in osteosarcoma. Cancer Res. 78 3938-3953 (2018).

128. Qin, Y. et al. Inhibition of histone lysine-specific demethylase 1 elicits breast tumor immunity and enhances antitumor efficacy of immune checkpoint blockade. Oncogene 38, 390-405 (2019).

129. Peng, D. et al. Epigenetic silencing of TH1-type chemokines shapes tumour immunity and immunotherapy. Nature 527, 249-253 (2015).

130. Boulding, T. et al. LSD1 activation promotes inducible EMT programs and modulates the tumour microenvironment in breast cancer. Sci. Rep. 8, 73 (2018).

131. Christmas, B. J. et al. Entinostat converts immuneresistant breast and pancreatic cancers into checkpoint-responsive tumors by reprogramming tumor-infiltrating MDSCs. Cancer Immunol. Res. 6 1561-1577 (2018).

132. Hicks, K . C et al. Epigenetic priming of both tumor and NK cells augments antibody-dependent cellular cytotoxicity elicited by the anti-PD-L1 antibody avelumab against multiple carcinoma cell types. Oncoimmunology 7, e1466018 (2018).

133. Degagné, E. et al. Tumor abscopal responses induced by the TLR9 agonist, SD-101, are strongly potentiated by a HDAC class I inhibitor, domatinostat. [abstract]. Cancer Res. 79 (Suppl 13), 2259 (2019).

134. Goswami, S. et al. Modulation of EZH2 expression in T cells improves efficacy of anti-CTLA-4 therapy. J. Clin. Invest. 128, 3813-3818 (2018).

135. Gounder, M. M. et al. Immunologic correlates of the abscopal effect in a SMARCB1/INI1-negative poorly differentiated chordoma after EZH2 inhibition and radiotherapy. Clin. Cancer Res. 25, 2064-2071 (2019).

136. Huang, S. et al. EZH2 inhibitor GSK126 suppresses antitumor immunity by driving production of myeloid-derived suppressor cells. Cancer Res. 79 2009-2020 (2019).

137. Saltos, A. N. et al. Phase I/lb study of pembrolizumab and vorinostat in patients with metastatic NSCLC (mNSCLC). J. Clin. Oncol. 36, 9046-9046 (2018).

138. Hellman, M et al. Efficacy/safety of entinostat (ENT) and pembrolizumab (PEMBRO) in NSCLC patients previously treated with anti-PD(L) 1 therapy. Presented at the 19th World Conference on Lung Cancer (2018).

139. Azad, N. S. et al. ENCORE 601: a phase 2 study of entinostat in combination with pembrolizumab in patients with microsatellite stable metastatic colorectal cancer. J. Clin. Oncol. 36, 3557-3557 (2018). 
140. Johnson, M. L. et al. ENCORE 601: a phase II study of entinostat (ENT) in combination with pembrolizumab (PEMBRO) in patients with melanoma. J. Clin. Oncol. 35, 9529-9529 (2017)

141. Levy, B. P. et al. Randomised phase 2 study of pembrolizumab plus CC-486 versus pembrolizumab plus placebo in patients with previously treated advanced non-small cell lung cancer. Eur. J. Cancer 108, 120-128 (2019).

142. Spigel, D. R. et al. Phase $1 / 2$ study of the safety and tolerability of nivolumab plus crizotinib for the first-line treatment of anaplastic lymphoma kinase translocation - positive advanced non-small cell lung cancer (CheckMate 370). J. Thorac. Oncol. 13, 682-688 (2018).

143. Khushalani, N. I. et al. A phase I trial of panobinostat with ipilimumab in advanced melanoma. J. Clin. Oncol. 35, 9547-9547 (2017).

144. Rodriguez, C. P. et al. Phase $\mathrm{I} / \mathrm{II}$ trial of pembrolizumab(P) and vorinostat $(\mathrm{V})$ in recurrent metastatic head and neck squamous cell carcinomas $(\mathrm{HN})$ and salivary gland cancer (SGC) [abstract]. J. Clin. Oncol. 36 (Suppl 15), 6025 (2018).

145. Bolden, J. E. et al. Inducible in vivo silencing of Brd4 identifies potential toxicities of sustained BET protein inhibition. Cell Rep. 8, 1919-1929 (2014).

146. Murphy, A. G. et al. Epigenetic priming prior to pembrolizumab in mismatch repair-proficient advanced colorectal cancer. J. Clin. Oncol. 37 591-591 (2019).

147. Grossman, S. R. p300/CBP/p53 interaction and regulation of the $\mathrm{p} 53$ response. Eur. J. Biochem. 268 2773-2778 (2001).

148. Karkhanis, V., Hu, Y.-J., Baiocchi, R. A., Imbalzano, A N. \& Sif, S. Versatility of PRMT5-induced methylation in growth control and development. Trends Biochem. Sci. 36, 633-641 (2011).

149. Dawson, M. A. The cancer epigenome: concepts, challenges, and therapeutic opportunities. Science 355, 1147-1152 (2017).

150. Campbell, R. M. \& Tummino, P. J. Cancer epigenetics drug discovery and development: the challenge of hitting the mark. J. Clin. Invest. 124, 64-69 (2014).

151. Le Loarer, F. et al. SMARCA4 inactivation defines a group of undifferentiated thoracic malignancies transcriptionally related to BAF-deficient sarcomas. Nat. Genet. 47, 1200-1205 (2015).

152. Hyman, D. M. et al. Vemurafenib in multiple nonmelanoma cancers with Braf $\mathrm{V} 600$ mutations. N. Engl. J. Med. 373, 726-736 (2015).

153. Zhong, J. et al. Enhanced and controlled chromatin extraction for chromatin-based epigenetic assays in FFPE tissues [abstract]. Cancer Res. 79 (Suppl 13), 5180 (2019)

154. Kim, K. H. et al. SWI/SNF-mutant cancers depend on catalytic and non-catalytic activity of EZH2. Nat. Med. 21, 1491-1496 (2015)

155. Smith-Roe, S. L. et al. SWI/SNF complexes are required for full activation of the DNA-damage response. Oncotarget 6, 732-745 (2015).

156. Rhyasen, G. W. et al. BRD4 amplification facilitates an oncogenic gene expression program in high-grade serous ovarian cancer and confers sensitivity to BET inhibitors. PLOS ONE 13, e0200826 (2018).

157. Wu, Y. et al. Therapeutic targeting of BRD4 in head neck squamous cell carcinoma. Theranostics 9 1777-1793 (2019).
158. McCabe, M. T. et al. EZH2 inhibition as a therapeutic strategy for lymphoma with EZH2-activating mutations. Nature 492, 108-112 (2012).

159. Corces, M. R. et al. An improved ATAC-seq protocol reduces background and enables interrogation of frozen tissues. Nat. Methods 14, 959-962 (2017).

160. Qu, K. et al. Chromatin accessibility landscape of cutaneous T cell lymphoma and dynamic response to HDAC inhibitors. Cancer Cell 32, 27-41 (2017).

161. Probst, A. V., Dunleavy, E. \& Almouzni, G. Epigenetic inheritance during the cell cycle. Nat. Rev. Mol. Cell Biol. 10, 192-206 (2009)

162. Shen, S. Y. et al. Sensitive tumour detection and classification using plasma cell-free DNA methylomes. Nature 563, 579-583 (2018).

163. Grosselin, K. et al. High-throughput single-cell ChIP-seq identifies heterogeneity of chromatin states in breast cancer. Nat. Genet. 51, 1060-1066 (2019).

164. Leroy, L. et al. Safety profile of epigenetic therapies in early phase trials: do epidrugs deserve specific drug development processes? [abstract 10P]. Ann. Oncol. 30 (Suppl 1), i4-i9 (2019).

165. Sivick, K. E. et al. Magnitude of therapeutic sting activation determines CD8+ T cell-mediated anti-tumor immunity. Cell Rep. 25, 3074-3085 (2018).

166. Champiat, S. et al. Hyperprogressive disease is a new pattern of progression in cancer patients treated by anti-PD-1/PD-L1. Clin. Cancer Res. 23, 1920-1928 (2017).

167. Gandhi, L. et al. Efficacy and safety of entinostat (ENT) and pembrolizumab (PEMBRO) in patients with non-small cell lung cancer (NSCLC) previously treated with anti-PD-(L) 1 therapy. J. Clin. Oncol. 36, 9036-9036 (2018).

168. Aggarwal, R. R. et al. Exceptional responders to abexinostat $(\mathrm{ABX})$ plus pazopanib (PAZ) in pretreated renal cell carcinoma (RCC) and other solid tumors: long-term follow-up of a phase $1 \mathrm{~b}$ study [abstract]. J. Clin. Oncol. 37 (Suppl.), 3022 (2019).

169. Dawson, M. et al. A phase I study of GSK525762, a selective bromodomain (BRD) and extra terminal protein (BET) inhibitor: results from part 1 of phase $\mathrm{I} / \mathrm{II}$ open label single agent study in patients with acute myeloid leukemia (AML). Blood 130, 1377-1377 (2017).

170. Fu, S. et al. Phase $1 \mathrm{~b}-2 \mathrm{a}$ study to reverse platinum resistance through use of a hypomethylating agent, azacitidine, in patients with platinum-resistant or platinum-refractory epithelial ovarian cancer. Cancer 117, 1661-1669 (2011).

171. Mazor, T., Pankov, A., Song, J. S. \& Costello, J. F. Intratumoral heterogeneity of the epigenome. Cancer Cell 29, 440-451 (2016).

172. Buenrostro, J. D. et al. Single-cell chromatin accessibility reveals principles of regulatory variation. Nature 523, 486-490 (2015)

173. Mohammad, F. \& Helin, K. Oncohistones: drivers of pediatric cancers. Genes Dev. 31, 2313-2324 (2017)

174. Rickman, D. S., Schulte, J. H. \& Eilers, M. The expanding world of N-MYC-driven tumors. Cancer Discov. 8 150-163 (2018).

\section{Acknowledgements}

The work of D.M. is funded by an Institut National de la Santê et de la Recherche Médicale (INSERM) l'Institut Thématique Multi-Organisme Cancer (ITMO Cancer) grant. The work of D.J. is funded in part by the Fondation ARC pour la Recherche sur le Cancer and Labex DEEP. The work of G.A. is funded by grants from la Ligue Nationale Contre le Cancer (Equipe labellisée Ligue), l'Agence Nationale de la Recherche (ANR-11LABX-0044 'DEEP', ANR-10-IDEX-0001-02 'PSL', ANR-12-BSV5-0022-02 'CHAPINHIB', ANR-14-CE16-0009 'Epicure', ANR-14-CE 10-0013 'CELLECTCHIP', ANR16-CE15-0018 'CHRODYT' and ANR-16-CE12-0024 'CHIFT') and the H2020 European Research Council (ERC-2015-POC project 678563 'EPOCH28' and ERC-2015-ADG- 694694 'ChromADICT'). The work of S.P.-V. is funded by the ATIPAvenir INSERM Young Group Leader grant programme and the Sites de Recherche Intégrée sur le Cancer (SIRIC: INCa-DGOS-INSERM_12551 'SOCRATE-2').

\section{Author contributions}

All authors made substantial contributions to all aspects of manuscript preparation. D.M. and D.J. contributed equally as co-first authors and G.A. and S.P.-V. contributed equally as co-supervisors.

\section{Competing interests}

As part of the Drug Development Department (DITEP), S.P.-V. has been a principal/sub-investigator of clinical trials for Aduro Biotech, Agios Pharmaceuticals, Amgen, Argen-X, Arno Therapeutics, Astex Pharmaceuticals, AstraZeneca, Aveo, Bayer, Beigene, Bioalliance Pharma, Biontech, Blueprint Medicines, Boehringer Ingelheim, Bristol-Myers Squibb, Celgene, Chugai Pharmaceutical, Clovis Oncology, Daiichi Sankyo, Debiopharm, Eisai, Exelixis, Forma, Gamamabs, Genentech, Gilead Sciences, GlaxoSmithKline, Glenmark Pharmaceuticals, H3 Biomedicine, Hoffmann-La Roche, Incyte, Innate Pharma, Iris Servier, Janssen, Kura Oncology, Kyowa Kirin, Lilly, Loxo Oncology, Lytix Biopharma, Medlmmune, Menarini Ricerche, Merck Sharp \& Dohme-Chibret, Merrimack Pharmaceuticals, Merus, Millennium Pharmaceuticals, Nanobiotix, Nektar Therapeutics, Novartis Pharma, Octimet Oncology, Oncoethix, Oncomed, Oncopeptides, Onyx Therapeutics, Orion Pharma, Oryzon Genomics, Pfizer, PharmaMar, Pierre Fabre, Rigontec, Roche, Sanofi Aventis, Sierra Oncology, Taiho Pharma, Tesaro, Tioma Therapeutics, to-BBB Technologies BV and Xencor. S.P.-V. has received research grants from AstraZeneca, Bristol-Myers Squibb, Boehringer Ingelheim, Janssen Cilag, Merck, Novartis, Pfizer, Roche and Sanofi, as well as non-financial research support (provision of drugs) from Astrazeneca, Bayer, Bristol-Myers Squibb, Boehringer Ingelheim, Johnson \& Johnson, Lilly, Medlmmune, Merck, NH TherAGuiX, Pfizer and Roche. S.P.-V. has also received research funding from Boehringer Ingelheim, Merck and Roche for research projects unrelated to this manuscript. S.P.-V. has participated on advisory boards for Merck and has benefited from non-financial support for attending symposia (travel paid and congress registration) from AstraZeneca. D.M., D.J., S.A. and G.A. declare no competing interests.

\section{Peer review information}

Nature Reviews Clinical Oncology thanks E. Choy, C. Jeronimo and the other, anonymous, reviewer for their contribution to the peer review of this work

\section{Publisher's note}

Springer Nature remains neutral with regard to jurisdictional claims in published maps and institutional affiliations.

\section{Supplementary information}

supplementary information is available for this paper at https://doi.org/10.1038/s41571-019-0267-4. 